\title{
Exponential basis functions in solution of incompressible fluid problems with moving free surfaces
}

S.M. Zandi

B. Boroomand

S. Soghrati 


\section{Exponential basis functions in solution of incompressible fluid problems with moving free surfaces problems with moving free surfaces}
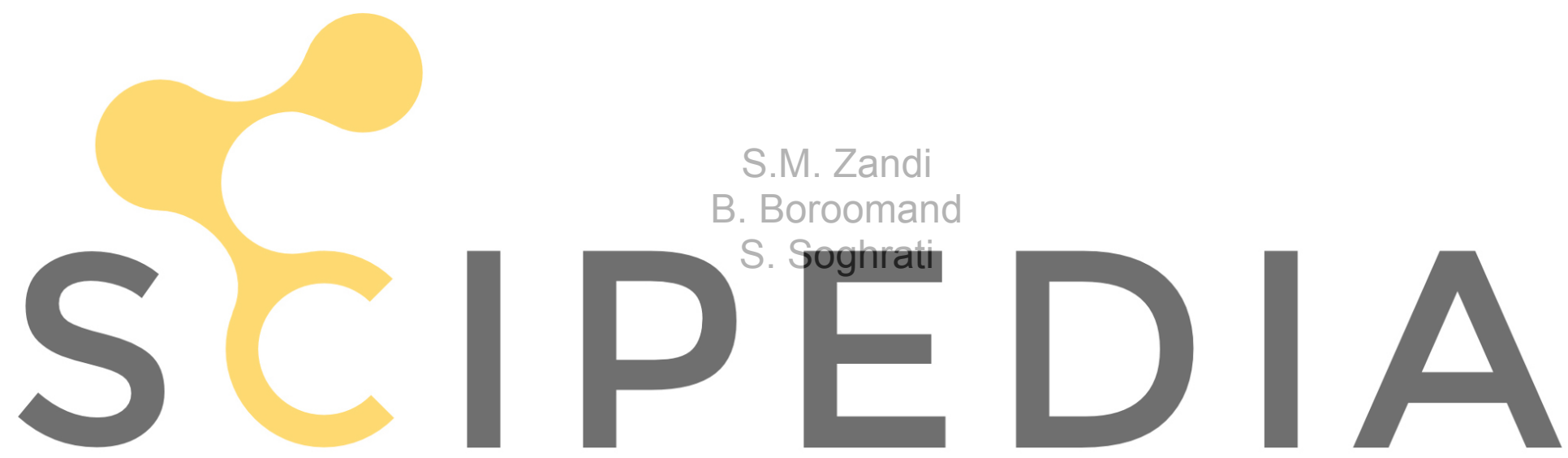

Register for free at https//www.scipedia.com to download the version without the watermark Publication CIMNE Nº-363, July 2011 


\section{Exponential basis functions in solution of incompressible fluid problems with moving free surfaces}

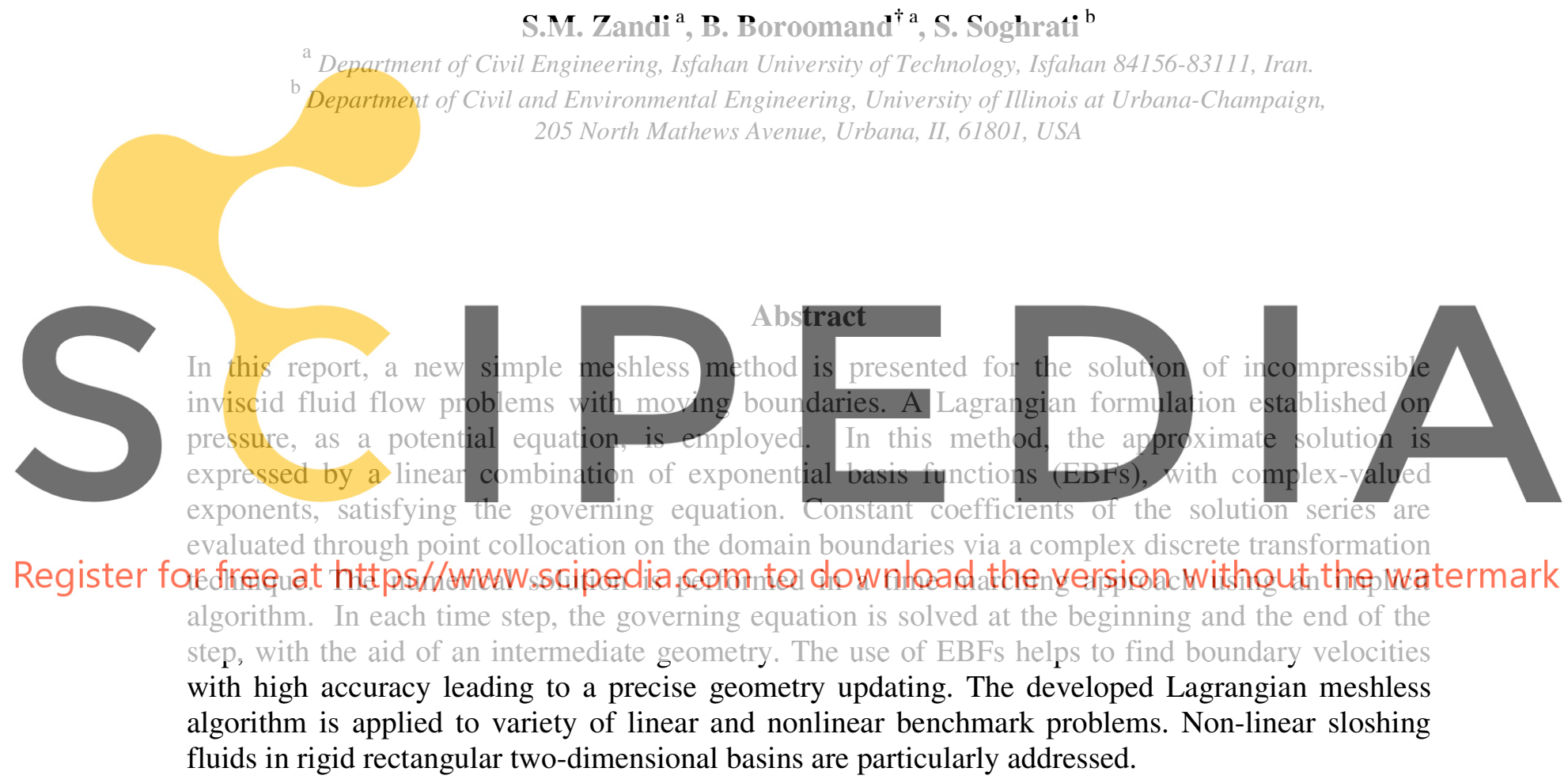

Keywords: free surface; moving boundaries; Lagrangian formulation; Trefftz method; exponential basis functions; meshless method; fluid-structure interaction

\footnotetext{
† Corresponding Author; Professor in Civil Engineering, Email: boromand@cc.iut.ac.ir
} 


\section{Contents}

1. Introduction

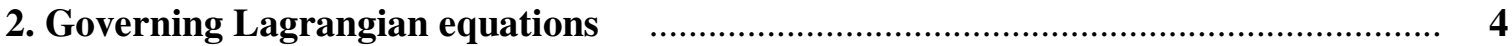

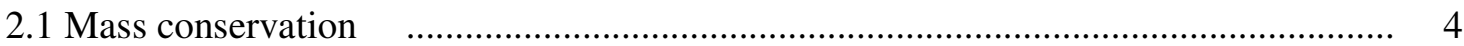

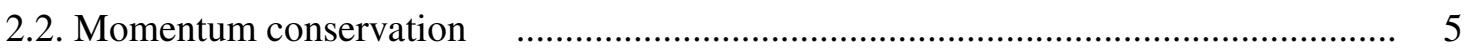

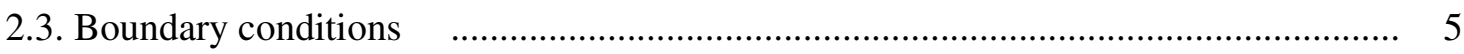

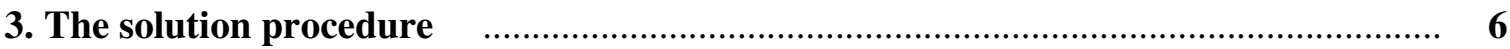

3.1. Spatial discretization of Laplace equation by the use of EBFs $\quad$.............................. 6

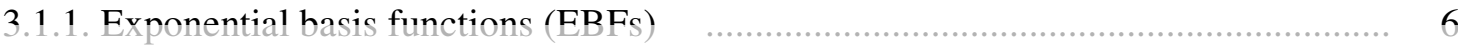

3.1,2. Imposition of boundary conditions using a discrete transformation $\quad$.................... 7

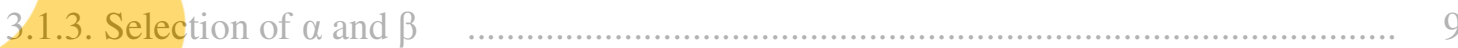

3.2. The time splitting and the geometry updating $\quad$...................................................... 9

3.3. Error indicator and regenerating boundary points $\quad$.................................................... 11

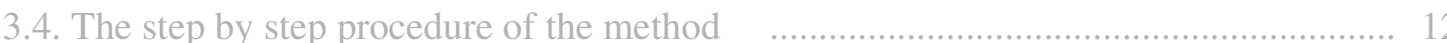

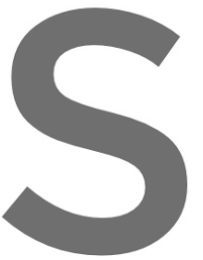

4. Numerical results

4.1. Water container under harmonic excitati

4.2. Standing wave in a rectangular tank

4.3. Large amplitude water oscillation in
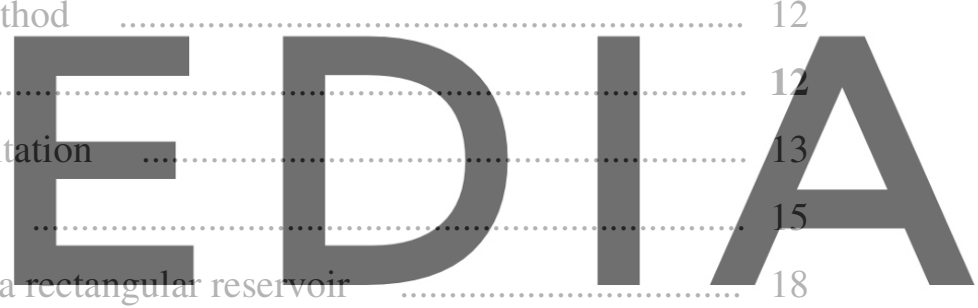

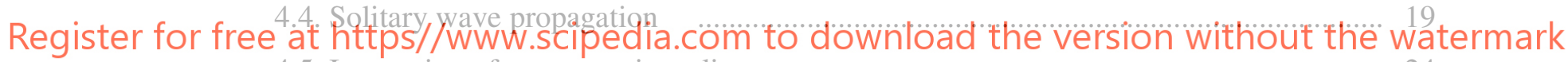
4.5. Interaction of two opposite solitary waves

\section{4}

5. Conchusions

24

Appendix A

26

References

26

List of Figures

28

List of Tables 


\section{INTRODUCTION}

Modelling of free surface fluid flow with moving boundaries has several applications in engineering problems such as the simulation of dam-reservoir interaction, liquid containing tanks subjected to earthquake, mould filling. The continuous variation of the shape of the domain with time during the numerical solution of such problems is a challenging issue. The main problem is thus the determination of the free surface position in order to update the geometry by considering the available numerical data.

Two fundamental approaches are generally implemented for the numerical solution of transient problems via a grid of points, e.g. in finite element method. The first is the use of Eulerian description, which treats the grid as a fixed computational reference and allows the fluid to move through it. The second approach is the Lagrangian description, in which the computational grid is embedded in the fluid and moves with it. Over the past thirty years, majority of the fluid flow simulations have been performed based on the Eulerian description [1]. However, methods based on the Lagrangian description have recently received more attention especially for solving free surface flow problems considering interaction with structures [2-5].

An intermediate approach is also sought in which the grid points are allowed to move independently of the fluid motion. This approach is named as arbitrary Lagrangian-Eulerian (ALE) method, and may be considered as a merged Lagrangian and Eulerian method for fluid flow. ALE has been widely used in conjunction with finite element method (FEM) for solving free surface flow problems [6-10].

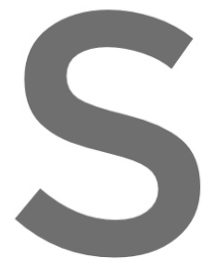

Rigorous and unavoic problems involving moviting adaptive meshing not but also for improving th major drawback of this r Another classification of th

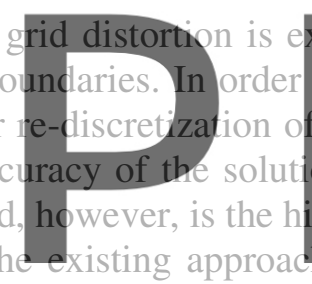
xpected when Lagrangian methods are
to avoid this problem, some researcher
f the fluid domain according to the new
tion (see e.g. [11] and the references the
high computational cost imposed to the prob
ches for addressing free surface flow pr applied to
geometry
problen.
problems is according to the necessity of using a grid in the computational procedure; e.g. mesh-based,

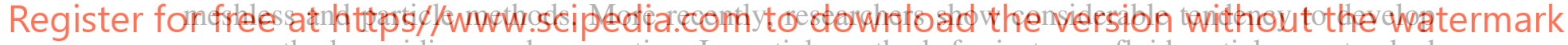
new methods avoiding mesh generation. In particle methods for instance, fluid particles are tracked using the Lagrangian description. The idea is traceable in the method proposed by Gingold and Monaghan [12] for the study of astrophysical hydrodynamic problems, known as Smooth Particle Hydrodynamics (SPH). This idea has been further developed and generalized for the solution of fluid mechanic problems [13-16]. On a similar basis, several computational methods using grids of points, known generally as meshless method, have so far been developed for fluid mechanic problems and sloshing phenomenon (see [17-19] for instance).

In the current work, a new simple method using the Lagrangian description is presented for the solution of a class of engineering problems involving incompressible inviscid fluid flow with moving boundaries interacting with rigid structures (weak interaction). In this method, the solution is expressed as a linear combination of exponential basis functions (EBFs), with complex-valued exponents, which satisfy the governing equations. Constant coefficients of the solution series are evaluated through point collocation on the domain boundaries via a complex discrete transformation technique. A time marching algorithm is used to solve the non-linear problems. Hence, in each time step, with the use of EBFs the pressure Laplace equation is solved at the beginning and end of the step, with the aid of an intermediate geometry. Other fluid variables such as acceleration, velocity and displacement are calculated accordingly. The geometry is then updated based on the Lagrangian formulation of motion through an implicit algorithm.

The transformation, proposed in [20-22], allows us to use a larger number of basis functions compared with the number of boundary information. The method has been applied to static and time 
harmonic elastic problems and described in more details in [23]. Also, in [24] the authors have recently used the method for analyzing fully incompressible elastic materials and incompressible steady Stokes flow in different domain shapes. Here, the developed Lagrangian meshless algorithm is applied to variety of linear and nonlinear benchmark problems such as liquid sloshing in rectangular basins, solitary wave propagation, etc.

With the use of basis functions satisfying the Laplace equation, the proposed method falls in the category of Trefftz methods (see [25] for a good review). However, the application of Trefftz method in problems with moving boundaries has not been previously reported in the literature.

The layout of the report is as follows. In Section 2 the model used for incompressible inviscid fluid flow is given. The solution procedure is explained in Section 3 which includes the definition of EBFs for the Laplace equation and the method of using the complex discrete transformation technique for imposition of the boundary conditions. We shall give a step-by-step summary of the procedure in the same section. In section 4, some benchmark problems are solved and the numerical results are compared with those obtained from analytical methods and other numerical approaches to show the capability of the proposed procedure.

\section{GOVERNING LAGRANGIAN EQUATIONS}

We consider an incompressible inviscid Newtonian fluid occupying a 2D bounded domain $\Omega$ with the boundaries as $\partial \Omega=\Gamma_{\mathrm{F}} \cup \Gamma_{\mathrm{S}}$. Here, $\Gamma_{\mathrm{F}}$ represents the free surface and $\Gamma_{\mathrm{S}}$ indicates the fluid-
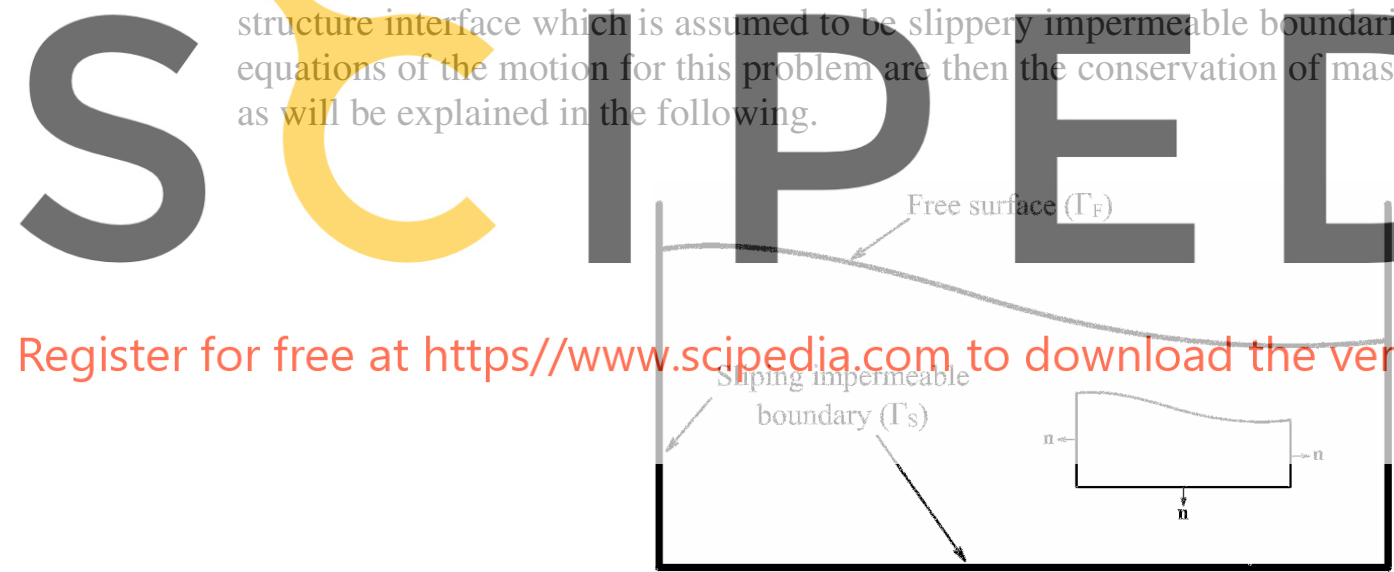

Figure 1. Problem domain and its boundaries

\subsection{Mass conservation}

The mass conservation equation in its general form can be written as

$\frac{\mathrm{D} \rho}{\mathrm{D} t}+\rho \nabla \cdot \mathbf{u}=0 \quad$ in $\Omega$

in which $\rho$ is the density and $\mathbf{u}$ is a vector containing the Cartesian components of the velocity field. Also $\mathrm{D} / \mathrm{D} t$ denotes the total or material derivative of the quantity and $\nabla$ is the well known gradient operator. This equation for an incompressible fluid with constant density may be written as

$\nabla . \mathbf{u}=0$.

The above equation is usually referred to as "incompressibility condition". 


\subsection{Momentum conservation}

The momentum conservation equation in its general form is expressed as

$\rho \frac{\mathrm{D} \mathbf{u}}{\mathrm{D} t}=-\nabla p+\mu \nabla^{2} \mathbf{u}+\frac{\mu}{3} \nabla(\nabla . \mathbf{u})+\rho \mathbf{g} \quad$ in $\Omega$.

In the above equation, $p$ is the pressure and $\mu$ is the fluid dynamic viscosity. The vector $\mathbf{g}=\left[\begin{array}{ll}0 & -g\end{array}\right]^{\mathrm{T}}$ is the source term vector including the gravity. The operator $\nabla^{2}$ is the Laplacian operator. For an incompressible fluid considering (2) we can write

$\rho \frac{\mathrm{D} \mathbf{u}}{\mathrm{D} t}=-\nabla p+\mu \nabla^{2} \mathbf{u}+\rho \mathbf{g}$,

and for inviscid fluid that $\mu=0$ we have

$\rho \frac{\mathrm{D} \mathbf{u}}{\mathrm{D} t}=-\nabla p+\rho \mathbf{g}$

The above equation is known as the Euler equation and should be solved by considering (2) as a constraint. However, by inner product of the gradient operator to both sides of (5), considering again the incompressibility condition (2), the above equation is simplified as follows

$\nabla^{2} p=0$ in $\Omega$.

Now, instead of (5) a

equations. To solve

boundary conditions

conditions for (6) are

or by prescribing the
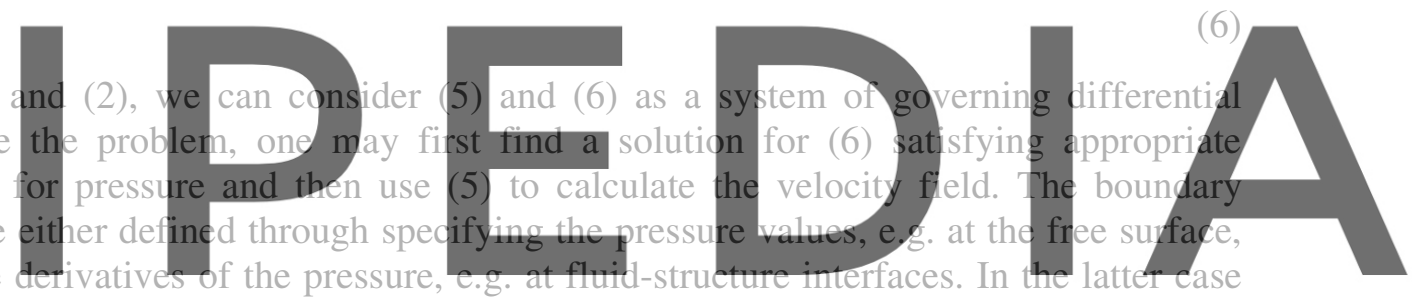

(5) is reused to define the conditions from information available for acceleration of the fluidRegister fortifuceereathththoss//www.scipedia.com to download the version without the watermark

\subsection{Boundary conditions}

In this subsection we express the fluid boundary conditions in terms of pressure. To this end we assume pressure as

$p=p_{H}-\rho g y$,

where $y$ is the vertical coordinate. Introducing (7) in (6) results in the following equation

$$
\nabla^{2} p_{H}=0 \text {. }
$$

This shows that $p_{H}$ in (7) is in fact the homogeneous part of the pressure field. Also by considering (7), Equation (5) takes the following form

$$
\rho \frac{\mathrm{Du}}{\mathrm{D} t}=-\nabla p_{H},
$$

which can be used in place of (5) when (8) is considered in place of (6). Considering $\mathbf{n}$ as the outward vector normal to the fluid boundaries (Fig. 1), the boundary condition at the fluid-structure interface, $\Gamma_{\mathrm{S}}$, is obtained by calculating the inner product of $\mathbf{n}$ with (9). This leads to the following relation 
$\frac{\partial p_{H}}{\partial n}=-\rho \mathbf{n}^{\mathrm{T}} \dot{\mathbf{u}}_{s} \quad$ on $\Gamma_{\mathrm{S}}$

where $\dot{\mathbf{u}}_{s}=D \mathbf{u}_{s} / D t$ denotes the predefined acceleration at the solid boundaries. At the free surface $\Gamma_{\mathrm{F}}$, the pressure should be equal to atmospheric pressure which is considered constant not only on the surface but also throughout the domain. Therefore, one may define the pressure as the difference between the actual fluid pressure and the atmospheric one and simply write the following condition

$p=0$ on $\Gamma_{\mathrm{F}}$.

By considering (7) the above equation may be rewritten as

$$
p_{H}=\rho g y \text { on } \Gamma_{\mathrm{F}}
$$

which plays the role of boundary condition at the free surface.

\section{THE SOLUTION PROCEDURE}

In this section, first the meshless method using EBFs for spatial discretization of Laplace equation is described and then the implicit time integration and geometry updating will be explained.

\subsection{Spatial discretization of Laplace equation by the use of EBFs}
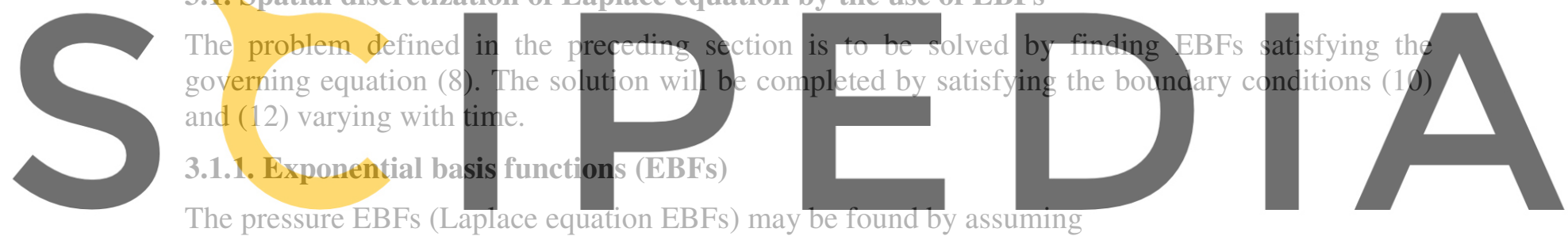

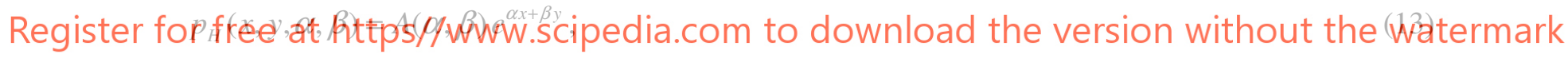

where $x$ and $y$ are the coordinates of a generic point in $\Omega, \alpha$ and $\beta$ are the complex valued exponents and $A(\alpha, \beta)$ is a constant independent of the coordinates. By inserting (13) in (8), the following equation is obtained

$$
\alpha^{2}+\beta^{2}=0 \text {. }
$$

From the above characteristic equation one may find $\alpha$ in terms of $\beta$ or vice versa as follows

$$
\alpha= \pm \mathbf{i} \beta \text { or } \beta= \pm \mathbf{i} \alpha \text {. }
$$

In which $\mathbf{i}=\sqrt{-1}$. With the relation obtained for the exponents, e.g. for $\alpha= \pm \mathbf{i} \beta$, one can write

$$
p_{H}=\int_{\Omega_{\beta}}\left\{A_{1}(\beta) e^{+\mathbf{i} \beta x+\beta y}+A_{2}(\beta) e^{-\mathbf{i} \beta x+\beta y}\right\} d \Omega_{\beta},
$$

In the above relation $\Omega_{\beta}$ is an appropriate area or locus on the Gaussian plane. The unknown coefficients $A_{1}(\beta)$ and $A_{2}(\beta)$ are to be found so that the boundary conditions (10) and (12) are satisfied. This is a very difficult task in many problems and therefore one may think of a discrete form of (16), for instance when the integral is to be calculated numerically, and simply write 


$$
\hat{p}_{H}=\sum_{i}\left(c_{i}^{1} e^{+\mathbf{i} \beta_{i} x+\beta_{i} y}+c_{i}^{2} e^{-\mathbf{i} \beta_{i} x+\beta_{i} y}\right)
$$

In the above equation $\hat{p}_{H}$ is an approximation to $p_{H}, c_{i}^{1}$ and $c_{i}^{2}$ represent a set of coefficients to be found from the boundary conditions and the summation is taken for the total number of points used on Gaussian plane for $\beta$. Similar relation can be written when $\beta_{i}= \pm \mathbf{i} \alpha_{i}$. In these series, $\alpha_{i}$ and $\beta_{i}$ are complex valued exponents that control the amplitude and fluctuations of the basis functions in the domain. Considering all cases, one may write the solution series in a general form as follows

$$
\hat{p}_{H}=\sum_{j=1}^{N} c_{j} e^{\alpha_{j} x+\beta_{j} y},
$$

in which $N$ is the total number of selected EBFs to achieve adequate accuracy. Note that in the above expression the EBFs obtained from calculating $\alpha_{j}$ in terms of $\beta_{j}$ and vice versa are included. It should also be noted that $\alpha_{i}$ and $\beta_{j}$ in (18) are related via the characteristic equation (14). Worthwhile to mention that when we calculate pressure gradients to evaluate fluid acceleration and velocity fields, the obtained velocity field satisfies the incompressibility condition (2) and therefore the incompressibility of fluid is directly fulfilled.
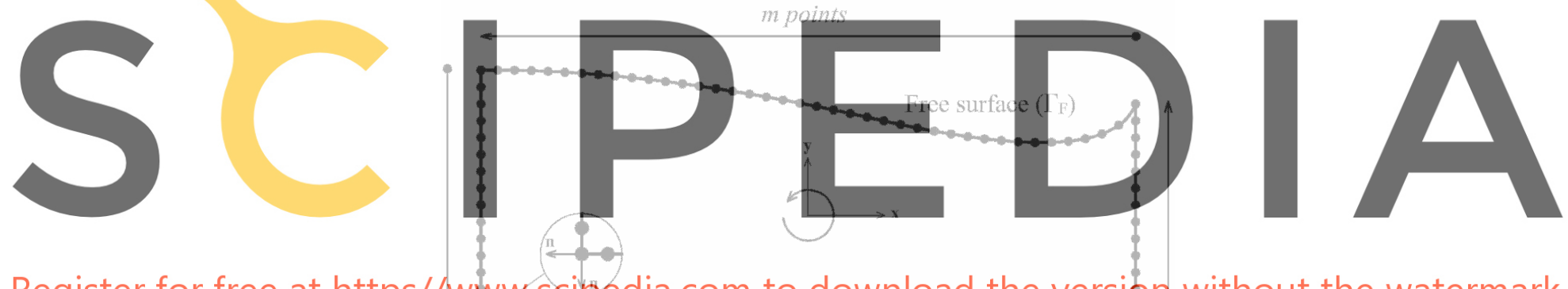

Register for free at https//
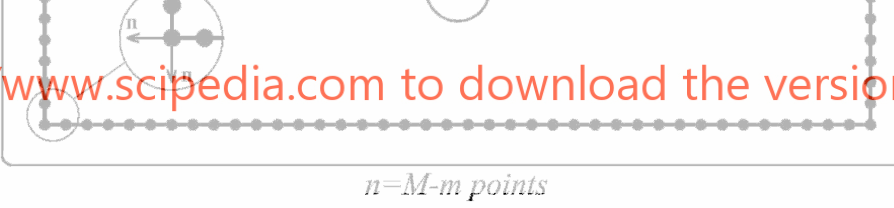

Figure 2. The boundary points used for the collocation approach

\subsubsection{Imposition of boundary conditions using a discrete transformation}

In this section, we shall follow the method proposed in [20-24] for the imposition of the boundary conditions. To this end, by considering $\hat{p}_{H}$ in its general form (18) and applying a collocation approach at $M$ boundary points one may write the following relation

$$
\overline{\mathbf{P}}_{\mathrm{B}}=\sum_{j=1}^{N} C_{j} \mathbf{V}_{j}
$$

In the above relation, $\overline{\mathbf{P}}_{\mathrm{B}}$ is a vector containing all point-wise boundary conditions, $\mathbf{V}_{j}$ is a normalized vector containing the contribution of each exponential basis to the boundaries arranged in the same manner as $\overline{\mathbf{P}}_{\mathrm{B}}$, and $C_{j}$ is proportional to the coefficient $c_{j}$ in (18) considering the normalization factor used in $\mathbf{V}_{j}$. For instance supposing that $m$ boundary points, out of $M$, are allocated to free surface $\Gamma_{\mathrm{F}}$ (Fig. 2) with prescribed pressure and $n=M-m$ points are allocated to fluid-structure interface $\Gamma_{\mathrm{S}}$ with prescribed acceleration (note that at the corners we choose two 
close points to define distinct normal vectors), then the vector $\overline{\mathbf{P}}_{\mathrm{B}}$ can be arranged in the following form:

$$
\overline{\mathbf{P}}_{\mathrm{B}}=\left\{\begin{array}{llllllll}
\left(p_{B}\right)_{1} & \left(p_{B}\right)_{2} & \ldots & \left(p_{B}\right)_{m} & \left(\partial p_{B}\right)_{1} & \left(\partial p_{B}\right)_{2} & \ldots & \left(\partial p_{B}\right)_{n}
\end{array}\right\}^{\mathrm{T}},
$$

where

$$
\left(p_{B}\right)_{k}=\left[\begin{array}{lll}
\rho g & y
\end{array}\right]_{x=x_{k}, y=y_{k}} \forall\left(x_{k}, y_{k}\right) \in \Gamma_{\mathrm{F}}, k=1, \ldots, m,
$$

and

$$
\left(\partial p_{B}\right)_{k}=-\rho\left[n_{x} \dot{u}_{x}+n_{y} \dot{u}_{y}\right]_{x=x_{k}, y=y_{k}} \quad \forall\left(x_{k}, y_{k}\right) \in \Gamma_{\mathrm{s}}, k=1, \ldots, n .
$$

In a similar manner $\mathbf{V}_{j}$ can be arranged as

$$
\mathbf{V}_{j}=\frac{1}{s_{i}}\left\{\begin{array}{llllllll}
\left(p_{j}\right)_{1} & \left(p_{j}\right)_{2} & \cdots & \left(p_{j}\right)_{m} & \left(\partial p_{j}\right)_{1} & \left(\partial p_{j}\right)_{2} & \cdots & \left(\partial p_{j}\right)_{n}
\end{array}\right\}^{\mathrm{T}},
$$

where $s_{j}$ is a scaling factor for normalization and

$$
\left(p_{j}\right)_{k}=\left[e^{\alpha_{j} x+\beta_{j} y}\right]_{x=x_{k}, y=y_{k}} \quad \forall\left(x_{k}, y_{k}\right) \in \Gamma_{\mathrm{F}}, k=1, \ldots, m
$$
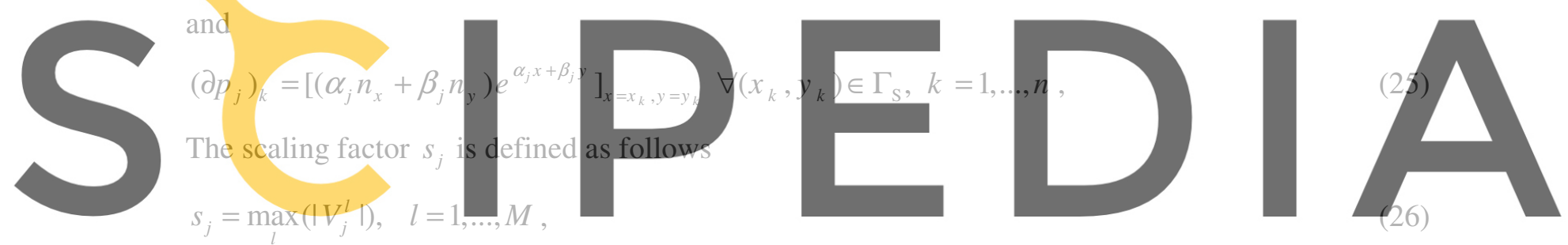

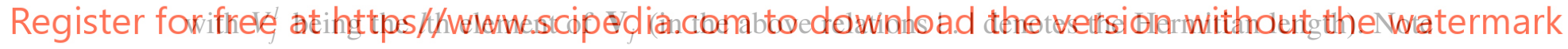

that with defining the scaling factor $s_{j}$, the coefficient $c_{j}$ in (18) is now related to $C_{j}$ in (19) as

$$
c_{j}=\frac{1}{s_{j}} C_{j} .
$$

Now for evaluating the coefficients $C_{j}$ we assume

$C_{j}=\mathbf{V}_{j}^{\mathrm{T}} \mathbf{R} \overline{\mathbf{P}}_{\mathrm{B}}$,

where $\mathbf{R}$ plays the role of a projection matrix assumed to be suitable for all $j=1, \ldots, N$. By inserting (28) in (19) we find

$$
\overline{\mathbf{P}}_{\mathrm{B}}=\sum_{j=1}^{N} \mathbf{V}_{j} \mathbf{V}_{j}^{\mathrm{T}} \mathbf{R} \overline{\mathbf{P}}_{\mathrm{B}}=\mathbf{G R} \overline{\mathbf{P}}_{\mathrm{B}}, \quad \mathbf{G}=\sum_{j=1}^{N} \mathbf{V}_{j} \mathbf{V}_{j}^{\mathrm{T}} .
$$

In the above relations, $\mathbf{G}$ is a symmetric $M \times M$ matrix. Since the rank of $\mathbf{G}$ might be less than $M$, $\mathbf{R}$ is evaluated as

$$
\mathbf{R}=\mathbf{G}^{+} \text {, }
$$


where $\mathbf{G}^{+}$is pseudo inverse of $\mathbf{G}$. Now we can evaluate $\hat{p}_{H}$ by the following equation

$$
\hat{p}_{H}=\Re\left[\left(\sum_{j=1}^{N} \frac{1}{s_{j}} e^{\alpha_{j} x+\beta_{j} y} \mathbf{V}_{j}^{\mathrm{T}}\right) \mathbf{R} \overline{\mathbf{P}}_{\mathrm{B}}\right] .
$$

In the above equation, $\mathfrak{R}[$.$] denotes the real part of the quantity. For further studies on the$ applications of the transformation used above the reader may refer to [20-23]. In view of (7), the pressure at any point of the fluid domain may be evaluated by

$$
\hat{p}=\mathfrak{R}\left[\left(\sum_{j=1}^{N} \frac{1}{s_{j}} e^{\alpha_{j} x+\beta_{j} y} \mathbf{V}_{j}^{\mathrm{T}}\right) \mathbf{R} \overline{\mathbf{P}}_{\mathrm{B}}\right]-\rho g y .
$$

Now a question may be cast on the way that $\alpha$ and $\beta$ are to be chosen.

\subsubsection{Selection of $\alpha$ and $\beta$}

The parameters $\alpha$ and $\beta$ play an important role in the variation of EBFs throughout the solution domain and thus have a prominent effect on the projection matrix $\mathbf{R}$. Since these parameters can take on complex values, we define a grid of points on Gaussian plane. The solution accuracy may differ by changing the grid. A detailed discussion on the selection of $\alpha$ and $\beta$ is out of the scope of this report and the reader may refer to [23] for further information. In this reference, the authors have suggested two simple strategies in this regard; one with mathematical basis and another heuristically based on numerical experiments. As shown in [23], the obtained accuracy with either of these approaches is satisfactory in all the cases studied. In this report we have used the heuristic strategy (see Appendix A). Note that the coefficients $\alpha_{j}, \beta_{j}$ are chosen once at the beginning of the solution, i.e. $t=0$, and are treated as constant coefficients during the time marching algorithm.

\subsection{The time splitting and the geometry updating}

Suppose that the total solution time has been divided into small increments. In the proposed algorithm here, the Laplace equation (8) should be solved twice at each time step. Assume that the solution is advanced to the $n$th time step $\left(\Delta t^{n}=t^{n+1}-t^{n}\right)$ and all static and kinematic variables from $t=0$ to $t=t^{n}$ are available. Let $\mathbf{x}^{n}$ and $\mathbf{u}^{n}=\mathbf{u}\left(\mathbf{x}^{n}, t^{n}\right)$ denote the current configuration and the current velocity, respectively. Vector of boundary conditions in the current configuration and time can then be evaluated using (20) in the following form

$\overline{\mathbf{P}}_{\mathrm{B}}^{n}=\overline{\mathbf{P}}_{\mathrm{B}}\left(\mathbf{x}^{n}, t^{n}\right)$.

Also for the contribution of the EBFs according to (23) we have

$$
\mathbf{V}_{j}^{n}=\mathbf{V}_{j}\left(\mathbf{x}^{n}, t^{n}\right) .
$$

The projection matrix $\mathbf{R}^{n}$ may be calculated as

$$
\mathbf{G}^{n}=\sum_{j=1}^{N} \mathbf{V}_{j}^{n} \mathbf{V}_{j}^{\mathrm{n}^{\mathrm{T}}}, \quad \mathbf{R}^{n}=\left(\mathbf{G}^{n}\right)^{+}
$$

Now $\hat{p}_{H}^{n}=\hat{p}_{H}\left(\mathbf{x}^{n}, t^{n}\right)$ can be evaluated using (31) as follows 


$$
\hat{p}_{H}^{n}=\mathfrak{R}\left[\left(\sum_{j=1}^{N} \frac{1}{s_{j}^{n}} e^{\alpha_{j} x+\beta_{j} y} \mathbf{V}_{j}^{n^{\mathrm{T}}}\right) \mathbf{R}^{n} \overline{\mathbf{P}}_{\mathrm{B}}^{n}\right] .
$$

Considering a as fluid acceleration vector, inserting (36) in (9) results in the following relation

$$
\mathbf{a}^{n}=\mathbf{a}\left(\mathbf{x}^{n}, t^{n}\right)=-\frac{1}{\rho} \mathfrak{R}\left[\left(\sum_{j=1}^{N} \frac{1}{s_{j}^{n}}\left\{\begin{array}{c}
\alpha_{j} \\
\beta_{j}
\end{array}\right\} e^{\alpha_{j} x+\beta_{j} y} \mathbf{V}_{j}^{n^{\mathrm{T}}}\right) \mathbf{R}^{n} \overline{\mathbf{P}}_{\mathrm{B}}^{n}\right] .
$$

By calculating acceleration at all boundary points, the new geometry $\tilde{\mathbf{x}}^{n+1}$, termed as intermediate configuration here, can be evaluated as

$$
\tilde{\mathbf{x}}^{n+1}=\mathbf{x}^{n}+\mathbf{u}^{n} \Delta t+\frac{1}{2} \mathbf{a}^{n} \Delta t^{2}
$$

We shall employ such a configuration for calculating the accelerations of the boundary points at the end of the time step. The coordinates given by (38) serve just as intermediate positions for the boundary points for calculating the boundary conditions to solve Laplace equation (8) at time $t=t^{n+1}$. In view of (20) and (33), the vector of boundary conditions for the intermediate configuration can be evaluated as

$\overline{\mathbf{P}}_{\mathrm{B}}^{n+1}=\overline{\mathbf{P}}_{\mathrm{B}}\left(\tilde{\mathbf{x}}^{n+1}, t^{n+1}\right)$.

Also for the vectors representing the contribution of EBFs

$$
\mathbf{V}_{j}^{n+1}=\mathbf{V}_{j}\left(\tilde{\mathbf{x}}^{n+1}, t^{n+1}\right) \text {. }
$$

The projection matrix is then obtained as

$$
\mathbf{G}^{n+1}=\sum_{j=1}^{N} \mathbf{V}_{j}^{n+1} \mathbf{V}_{j}^{\mathrm{n}+1^{\mathrm{T}}}, \quad \mathbf{R}^{n+1}=\left(\mathbf{G}^{n+1}\right)^{+}
$$

Now the acceleration vector can be evaluated in manner analogous to (37) as

$$
\mathbf{a}^{n+1}=-\frac{1}{\rho} \mathfrak{R}\left[\left(\sum_{j=1}^{N} \frac{1}{s_{j}^{n+1}}\left\{\begin{array}{c}
\alpha_{j} \\
\beta_{j}
\end{array}\right\} e^{\alpha_{j} x+\beta_{j} y} \mathbf{V}_{j}^{n+1^{\mathrm{T}}}\right) \mathbf{R}^{n+1} \overline{\mathbf{P}}_{\mathrm{B}}^{n+1}\right] .
$$

After calculating $\mathbf{a}^{n}=\mathbf{a}\left(\mathbf{x}^{n}, t^{n}\right)$ and $\mathbf{a}^{n+1}=\mathbf{a}\left(\tilde{\mathbf{x}}^{n+1}, t^{n+1}\right)$ at all boundary points, assuming linear acceleration within each time step, the final configuration $\mathbf{x}^{n+1}$ of boundary points at the end of step can be evaluated as

$$
\mathbf{x}^{n+1}=\mathbf{x}^{n}+\mathbf{u}\left(\mathbf{x}^{n}, t^{n}\right) \Delta t+\frac{1}{6}\left(2 \mathbf{a}\left(\mathbf{x}^{n}, t^{n}\right)+\mathbf{a}\left(\tilde{\mathbf{x}}^{n+1}, t^{n+1}\right)\right) \Delta t^{2} .
$$

The velocity field at the boundary points of the final configuration can be then calculated as

$$
\mathbf{u}^{n+1}=\mathbf{u}\left(\mathbf{x}^{n+1}, t^{n}\right)+\frac{1}{2}\left(\mathbf{a}\left(\mathbf{x}^{n+1}, t^{n}\right)+\mathbf{a}\left(\mathbf{x}^{n+1}, t^{n+1}\right)\right) \Delta t
$$

The new configuration may be regarded as a new intermediate one and the procedure may be repeated until a unique $\mathbf{x}^{n+1}$ is obtained, but our experience shows that excellent accuracy is 
achievable by just one step. Having calculated $\mathbf{x}^{n+1}$ and $\mathbf{u}^{n+1}$, one may use them as the initial position and velocity, respectively, of the boundary points at the beginning of the next time step.

\subsection{Error indicator and regenerating boundary points}

Similar to other boundary mesh based or meshless methods, here deviation of the numerical solution from the exact one may be understood by evaluating the discrepancy of the approximated boundary values from the exact ones [23]. It must be noted that since the EBFs satisfy the elliptic equation (8), i.e. there is no residual in the interior parts of the domain, the residuals of the boundary conditions can be employed as an appropriate error indicator. Considering the content of Section 3.1, an error indicator may be devised by the re-evaluation of the boundary collocated values. This means that by (31) in hand, one may find approximated vector of $\overline{\mathbf{P}}_{\mathrm{B}}$ named here as $\tilde{\overline{\mathbf{P}}}_{\mathrm{B}}$ so that

$\tilde{\overline{\mathbf{P}}}_{\mathrm{B}}=\left\{\begin{array}{llllllll}\left(\hat{p}_{B}\right)_{1} & \left(\hat{p}_{B}\right)_{2} & \cdots & \left(\hat{p}_{B}\right)_{m} & \left(\begin{array}{llll}\left.\partial \hat{p}_{B}\right)_{1} & \left(\partial \hat{p}_{B}\right)_{2} & \ldots & \left(\partial \hat{p}_{B}\right)_{n}\end{array}\right\}^{\mathrm{T}},\end{array}\right.$

where

$$
\begin{aligned}
& \left(\hat{p}_{B}\right)_{k}=\left[\hat{p}_{H}\right]_{x=x_{k}, y=y_{k}}, \\
& \left(\partial \hat{p}_{B}\right)_{k}=\left[\frac{\partial \hat{p}_{H}}{\partial n}\right]_{x=x_{k}, y=y_{k}} .
\end{aligned}
$$

By a collocation error defined as

$$
\tilde{\mathbf{e}}=\overline{\mathbf{P}}_{\mathrm{B}}-\tilde{\overline{\mathbf{P}}}_{\mathrm{B}}
$$

one may define an error norm as an indicator for the errors in the collocated boundary values. A similar error norm may be defined for other boundary points different from those used for the collocation. Such indicators are suitable for judging the performance of the transformation technique used in Section 3.1. The smallness of the error norms indicates the suitability of the EBFs and boundary points used.

In problems with large displacements where the surfaces deform considerably, the distribution of boundary points may become irregular after several time steps and thus the accuracy of the calculated pressure may decrease. This can be checked by evaluating the error norms in each time step as in (48). In such a case, by using a simple strategy we can regenerate regular boundary points to decrease such errors in the next time step. To alleviate this effect one may use a regularization technique for rearranging the boundary nodes. In this study, we suppose that the free surface profile between two adjacent boundary points, in Figure 2 for instance, at the end of the step is linear and can be approximated as

$$
y=\frac{y_{i+1}-y_{i}}{x_{i+1}-x_{i}}\left(x-x_{i}\right)+y_{i}
$$

where $\left(x_{i}, y_{i}\right)$ and $\left(x_{i+1}, y_{i+1}\right)$ are the coordinates of the two adjacent boundary points. Now we can easily generate new regular points with similar distances on free surface boundary. These new boundary points should be used as $\mathbf{x}^{n+1}$ in (44) for evaluating the initial velocities of the next step. This simple strategy does not impose remarkable computational cost and so in this research we regenerate the boundary points in each time step without evaluating the errors. 


\subsection{The step by step procedure of the method}

To give an insight into the implementation of the method, we present the step by step procedure. After defining the problem geometry,

1. Choose a series of points on the boundary of the domain. At corners choose two close points to define distinct normal vectors.

2. Initialize the velocities of the boundary points if the problem does not start at rest.

3. Choose a grid of points on Gaussian plane. The bounds of the values are preliminarily determined by considering the oscillation of the boundary values [23] (see Appendix A).

4. For each point of the grid in step 3, for instance when $\alpha_{i}= \pm \mathbf{i} \beta_{i}$, evaluate the set of EBFs as given in (17) and analogously proceed for when $\beta_{i}= \pm \mathbf{i} \alpha_{i}$.

5. Evaluate the collocated values of the boundary conditions in the current time and configuration in a vector as defined in (33), that is $\overline{\mathbf{P}}_{\mathrm{B}}^{n}=\overline{\mathbf{P}}_{\mathrm{B}}\left(\mathbf{x}^{n}, t^{n}\right)$.

6. For each EBF constructed in step 4, evaluate its contribution on the boundaries, that is $\mathbf{V}_{j}^{n}$.

7. Evaluate $\mathbf{G}^{n}=\sum_{j=1}^{N} \mathbf{V}_{j}^{n} \mathbf{V}_{j}^{\mathrm{n}^{\mathrm{T}}}$.

8. Evaluate $\mathbf{R}^{n}=\left(\mathbf{G}^{n}\right)^{+}$.

9. Evaluate the Lagrangian acceleration for each boundary points at the beginning of the step as given in (37).

10. Calculate the coordinates of the intermediate configuration $\tilde{\mathbf{x}}^{n+1}$ by (38).

11. Evaluate the collocated values of the boundary conditions at time $t=t^{n+1}$ and intermediate configuration in a vector as defined in (39), that is $\overline{\mathbf{P}}_{\mathrm{B}}^{n+1}=\overline{\mathbf{P}}_{\mathrm{B}}\left(\tilde{\mathbf{x}}^{n+1}, t^{n+1}\right)$.

12. For each EBF of step 4, evaluate its contribution on the new boundaries, that is $\mathbf{V}_{j}^{n+1}$.

13. Evaluate $\mathbf{G}^{n+1}=\sum_{j=1}^{N} \mathbf{V}_{j}^{n+1} \mathbf{V}_{j}^{\mathrm{n}+1^{\mathrm{T}}}$.

14. Evaluate $\mathbf{R}^{n+1}=\left(\mathbf{G}^{n+1}\right)^{+}$.

15. Evaluate the Lagrangian acceleration for each boundary points at the end of the step as given in (42).

16. Calculate the final position of the boundary points by (43). (One may consider this final configuration as a new intermediate configuration and repeat from 11 until the difference between the last two configurations become sufficiently small.)

17. Regenerate a set of new regular boundary points according to the free surface profile in the previous step and consider them as $\mathbf{x}^{n+1}$.

18. Calculate the velocities of the new boundary points through (44).

19. Set $\mathbf{x}^{n+1}$ and $\mathbf{u}^{n+1}$ as initial configuration and velocity of each boundary point for the next time step.

20. Repeat from 5 for the next time step.

\section{NUMERICAL RESULTS}

In this part we present the results of the method applied to some benchmark problems. Numerical results have been compared with those obtained from analytical methods and other numerical approaches to show the capability of the proposed procedure. 


\subsection{Water container under harmonic excitation}

We consider the problem of sloshing in a water container under harmonic excitation as the first numerical experiment. Sloshing effect is seen in many engineering applications such as liquid containing tanks or dams subjected to earthquake. Many numerical methods have so far been employed to solve sloshing problems, such as FEM [2], finite difference methods (FDM) [26], and boundary element methods (BEM) [27]. The main aim is to accurately calculate the hydrodynamic pressure which is considered a key-factor in designing of the structure. An extended history of the sloshing problems has been given in [28].

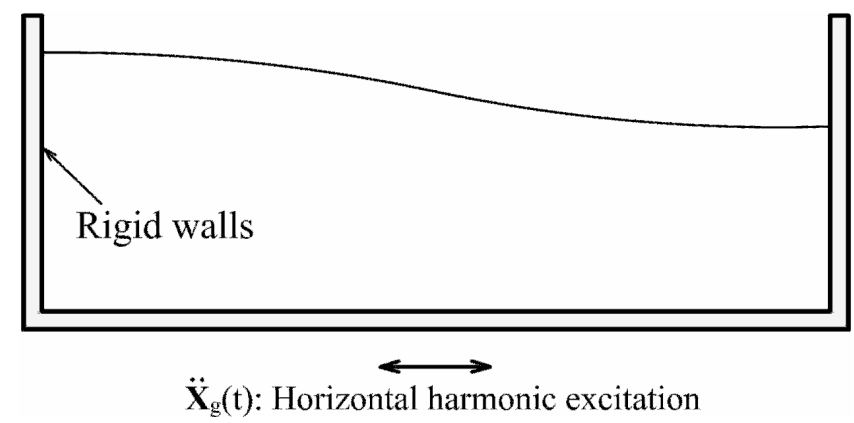

Figure 3. Water container under harmonic excitation (Example 4.1)

A rectangular rigid tank is considered as the numerical example (Fig. 3). The length of the container $L$ and the still water depth $h$ are $80 \mathrm{~cm}$ and $10 \mathrm{~cm}$, respectively. The tank width, $B$, for base shear calculation is $14.1 \mathrm{~cm}$. These parameters have been chosen from reference [27]. The water density and gravity acceleration are $\rho=1000 \mathrm{~kg} / \mathrm{m}^{3}$ and $g=9.81 \mathrm{~m} / \mathrm{s}^{2}$, respectively. According to linear wave theorem, the first natural frequency $\omega_{1}$ is as follows

$\omega_{1}=\sqrt{\frac{\pi g}{L} \tanh \frac{\pi h}{L}}$.

Hence, the first natural frequency of this reservoir is $3.79 \mathrm{rad} / \mathrm{s}$. For numerical modeling, 142 points are used on boundaries (38 points are on the left and right walls and 104 points are on the bottom and free surfaces (Fig. 4)). No regularization is used for the points in this problem. The number of EBFs used for this simulation is 400 .

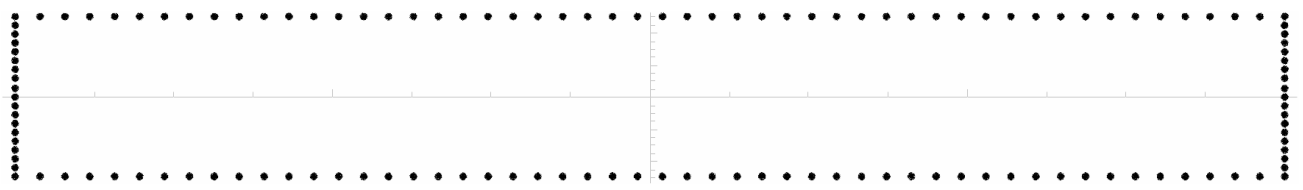

Figure 4. The boundary points used for water container in Example $4.1(\mathrm{~L}=80 \mathrm{~cm}, \mathrm{~h}=10 \mathrm{~cm})$

The tank is subjected to ground oscillation in the horizontal direction with the following acceleration

$\ddot{\mathrm{X}}_{\mathrm{g}}(t)=A_{\mathrm{f}} \omega_{\mathrm{f}}^{2} \cos \left(\omega_{\mathrm{f}} t\right)$.

In (51) $A_{\mathrm{f}}$ and $\omega_{\mathrm{f}}$ are, respectively, the displacement amplitude and the frequency of the harmonic excitation. 
For the evaluation of the base shear force $F_{b}$ we write

$$
F_{b}=\int_{\Gamma_{\mathrm{S}}} p_{x} \mathrm{~d} \Gamma_{\mathrm{S}},
$$

in which $p_{x}=p n_{x}$. Also, by calculating the difference of the hydrostatic forces at two sides of the lateral walls, the hydrostatic base shear force is calculated by the following equation (see also [27])

$$
\bar{F}_{b}=\frac{\rho g B}{2}\left(h_{R}^{2}-h_{L}^{2}\right) \text {. }
$$

In the above relation, $h_{R}$ and $h_{L}$ are the wave elevations on the right and left walls, respectively.

Here, the simulation has been carried out for harmonic ground motion with the amplitude of $A_{\mathrm{f}}=0.04 \mathrm{~cm}$ and frequencies of $1.9,3.79$ and $11.38 \mathrm{rad} / \mathrm{s}$. We have used $\Delta t=0.01 \mathrm{sec}$ for $\omega_{\mathrm{f}}=1.9$ $\mathrm{rad} / \mathrm{s}$ and $\Delta t=0.005 \mathrm{sec}$ for $\omega_{\mathrm{f}}=3.79$ and $11.38 \mathrm{rad} / \mathrm{s}$.
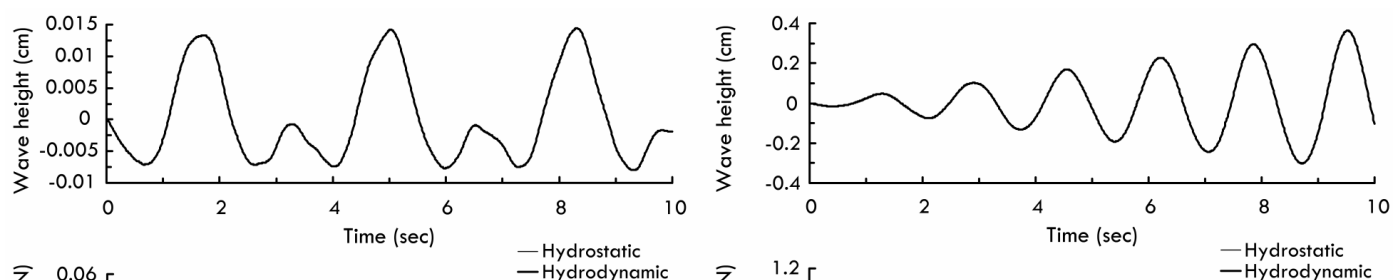

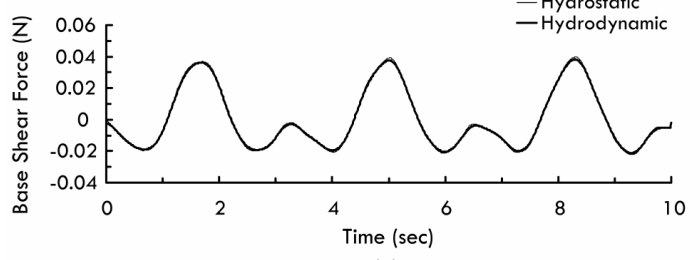

(a)

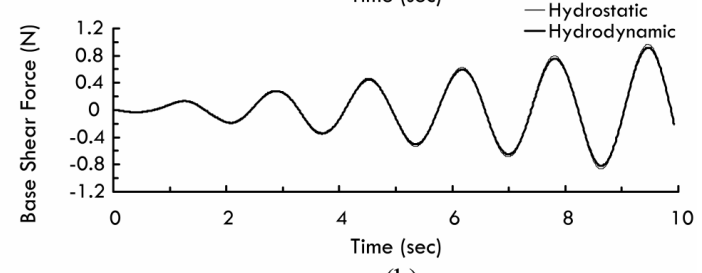

(b)
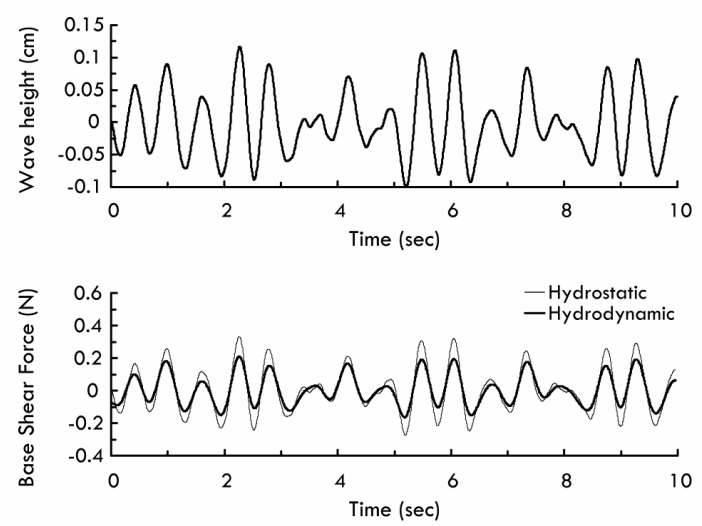

(c)

Figure 5. The results obtained for the rectangular tank (Example 4.1) subjected to harmonic excitation with forced frequency: (a) $\omega_{\mathrm{f}}=1.90 \mathrm{rad} / \mathrm{s},(\mathrm{b}) \omega_{\mathrm{f}}=3.79 \mathrm{rad} / \mathrm{s}$, (c) $\omega_{\mathrm{f}}=11.38 \mathrm{rad} / \mathrm{s}$.

Figure 5 demonstrates the results of the simulation which are in excellent agreement with those given in [27] obtained by BEM. The results are including the wave height at the right lateral wall and the base shear force. Free surface profiles of the rectangular tank subjected to harmonic excitation with forced frequency $\omega_{\mathrm{f}}=3.79 \mathrm{rad} / \mathrm{s}$ are shown in Figure 6. 

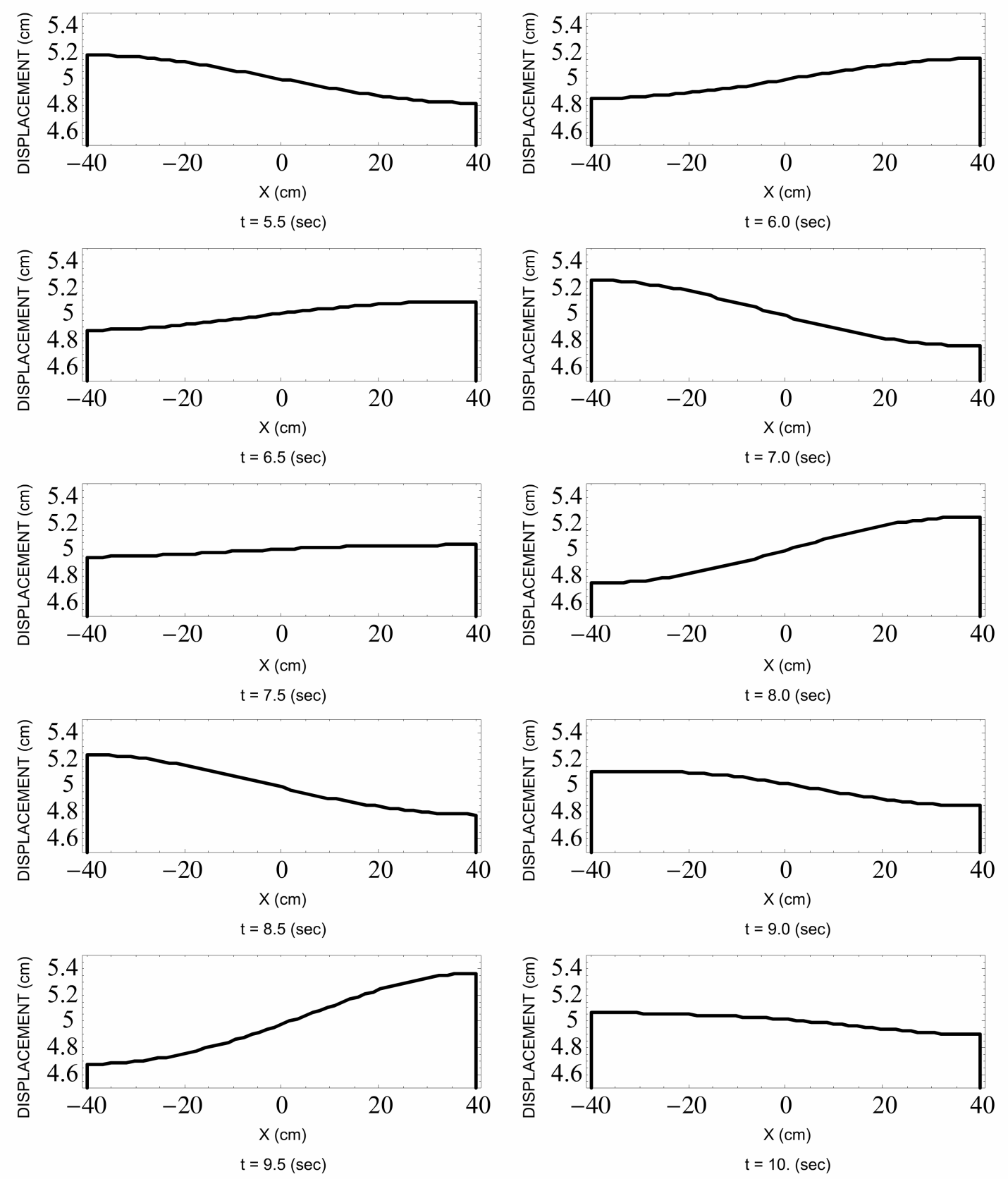

Figure 6. Free surface profiles of the rectangular tank (Example 4.1) subjected to harmonic excitation with forced frequency $\omega_{\mathrm{f}}=3.79 \mathrm{rad} / \mathrm{s}$ at different time steps.

\subsection{Standing wave in a rectangular tank}

Water oscillation with an initial free surface profile in a rectangular tank has been simulated in many references by different numerical methods $[2,4,8,14,16]$. Consider a rectangular rigid tank with a cosine wave at the free surface as the initial condition (Fig. 7). 


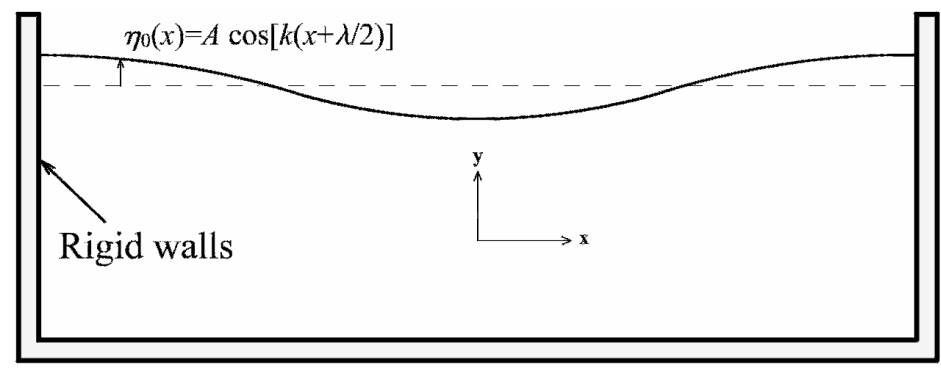

Figure 7. Standing wave problem (example 4.2).

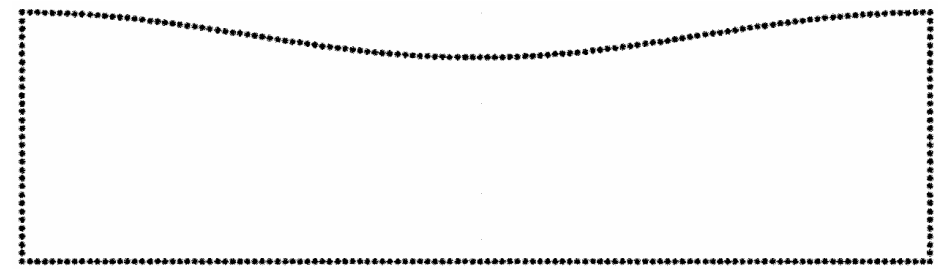

Figure 8. Initial free surface profile and boundary points used for standing wave problem (Example 4.2).

The initial free surface profile is given by

$\eta_{0}(x)=A \cos [k(x+\lambda / 2)]$,

where $\eta_{0}(x)$ is the initial free surface displacement, $A$ and $\lambda$ are the wave amplitude and the wavelength, respectively. Also, $k=2 \pi / \lambda$ is the wave number. An analytical solution has been given in [29] for the wave height at the center of the tank considering nonlinear effects. According to the solution, the wave elevation is the summation of a linear term as

$\eta_{1}(t)=-A \cos \left(\omega_{2} t\right)$

and a second order term as

$\eta_{2}(t)=\frac{1}{8 g}\left\{2\left(A \omega_{2}\right)^{2} \cos \left(2 \omega_{2} t\right)+\frac{A^{2}}{\omega_{2}^{2}}\left[k_{2}^{2} g^{2}+\omega_{2}^{4}-\left(k_{2}^{2} g^{2}+3 \omega_{2}^{4}\right) \cos \left(\omega_{4} t\right)\right]\right\}$,

where

$k_{n}=n \pi / \lambda$,

$\omega_{n}=\left[k_{n} g \tanh \left(k_{n} h\right)\right]^{1 / 2}$.

In this simulation we have used $\rho=1000 \mathrm{~kg} / \mathrm{m}^{3}$ and $g=9.81 \mathrm{~m} / \mathrm{s}^{2}$. The length of the reservoir is $L=\lambda=2 \mathrm{~m}$ and the depth of water is $h=0.5 \mathrm{~m}$ in the equilibrium state. The wave amplitude is considered as $A=0.1 \mathrm{~h}$. For modelling this problem, we have used 306 boundary points (see Fig. 8) and 400 EBFs. The time increment $\Delta t$ is chosen as $0.01 \mathrm{sec}$. In Figure 9, wave height at the center of the tank is depicted considering both the linear theory $\eta=\eta_{1}$ and the second order theory $\eta=\eta_{1}+\eta_{2}$. The results show the accuracy and capability of the method in simulating nonlinear effects. The hydrodynamic pressure, in Figure 10, is evaluated as 


$$
p_{\text {Hydrodynamic }}=p-\rho g h_{w} \text {. }
$$

in which $h_{w}$ is the water column height above the point under consideration in the fluid domain. As is seen in this Figure, the details of the pressure distribution are fully captured.

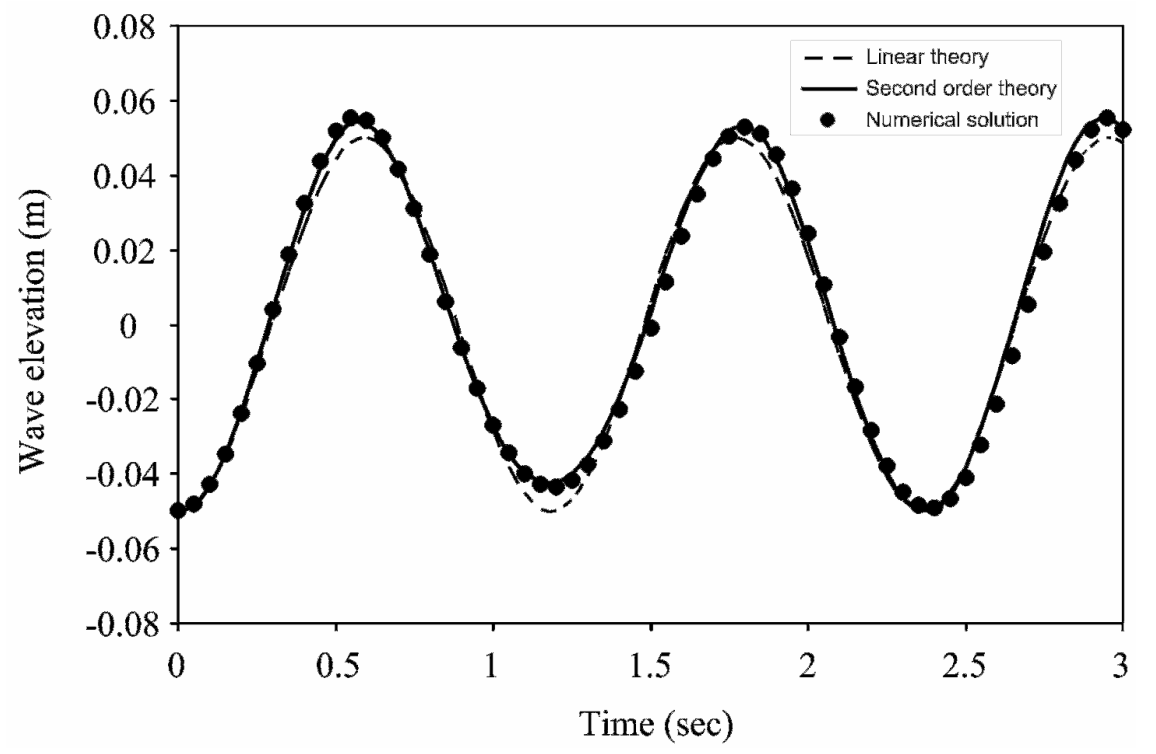

Figure 9. Comparison of the calculated wave heights at the center of the tank (Example 4.2).
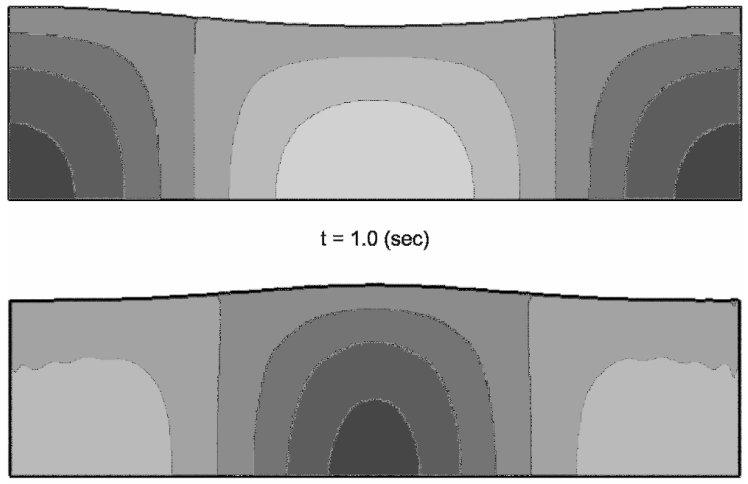

$\mathrm{t}=2.0(\mathrm{sec})$

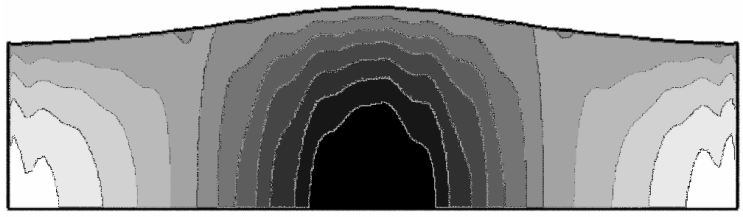

$\mathrm{t}=3.0(\mathrm{sec})$

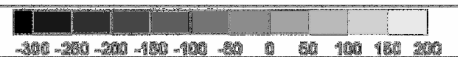

Figure 10. Hydrodynamic pressure (Pa) at different time steps (Example 4.2). 


\subsection{Large amplitude water oscillation in a rectangular reservoir}

As the next numerical experiment, we consider a rectangular basin with a half cosine wave at free surface (Fig. 11).

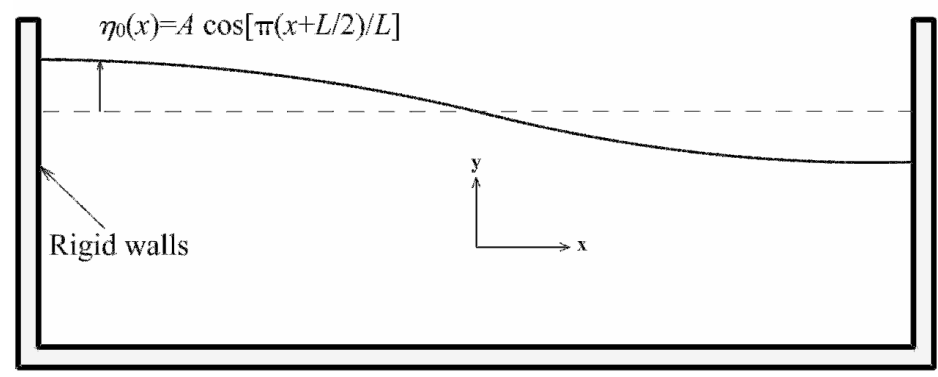

Figure 11. Rectangular basin with a half cosine wave (Example 4.3).

The initial free surface profile is given by

$\eta_{0}(x)=A \cos \left(\frac{\pi(x+L / 2)}{L}\right)$.

The definitions of parameters in (59) are the same as those in the previous example. According to shallow water theory [30], the fluid velocity field for small amplitude oscillations is as follows

$$
\begin{aligned}
& u(x, y, t)=\frac{A \sqrt{g h}}{h} \sin \left(\frac{\pi(x+L / 2)}{L}\right) \sin \left(\frac{\pi \sqrt{g h}}{L} t\right), \\
& v(x, y, t)=-\frac{A \sqrt{g h} \pi(y+h / 2)}{h L} \cos \left(\frac{\pi(x+L / 2)}{L}\right) \sin \left(\frac{\pi \sqrt{g h}}{L} t\right) .
\end{aligned}
$$

where $u$ and $v$ are the velocity components and $h$ is the still water depth. So the wave elevation at left and right walls and center of the basin may be evaluated by

$$
\begin{aligned}
& \eta_{\text {(left) }}(t)=A+\int_{0}^{t} v_{(-L / 2, h / 2, t)} d t, \\
& \eta_{\text {(center) }}(t)=\int_{0}^{t} v_{(0, h / 2, t)} d t, \\
& \eta_{\text {(right) }}(t)=-A+\int_{0}^{t} v_{(L / 2, h / 2, t)} d t .
\end{aligned}
$$

This problem has been solved in [8] using arbitrary Lagrangian-Eulerian finite element and velocity-vorticity formulation of fluid flow equations. In the reference, 4137 elements have been used and the time increment has been chosen as $\Delta t=0.02 \mathrm{sec}$ to simulate the problem for $L=98 \mathrm{~m}$ and $h=5 \mathrm{~m}$.

Here, we have used 294 boundary points and 400 EBFs for modelling. The time increment $\Delta t$ and all other parameters are the same as those used in [8]. This simulation has been carried out for different wave amplitudes. In Figures 12 and 13 the wave elevation for the wave amplitudes $A=0.1 \mathrm{~m}$ and $A=0.5 \mathrm{~m}$ are shown. The results are in excellent agreement with those given in [8]. By increasing the initial free surface wave amplitude, the difference between linear analytical solution and nonlinear numerical analysis significantly grows. 


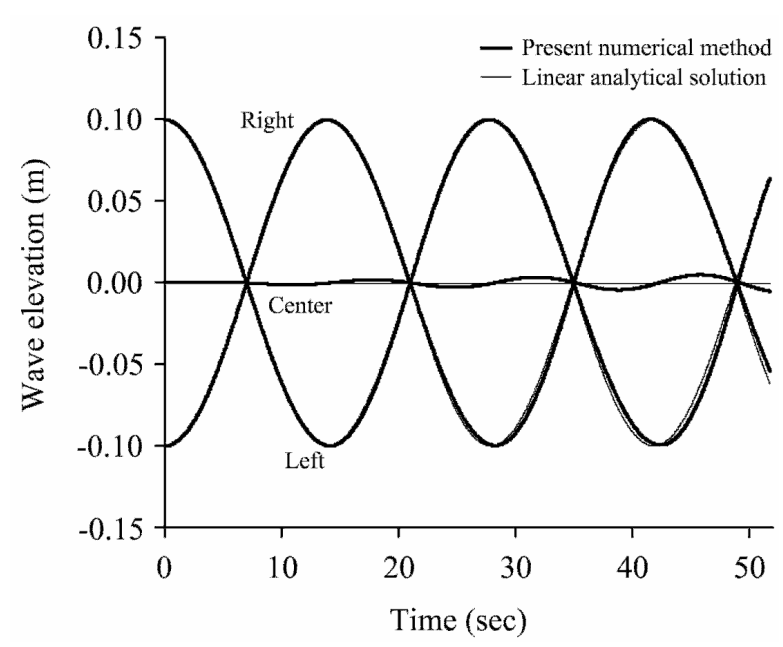

Figure 12. Surface wave elevation for $0.1 \mathrm{~m}$ amplitude.

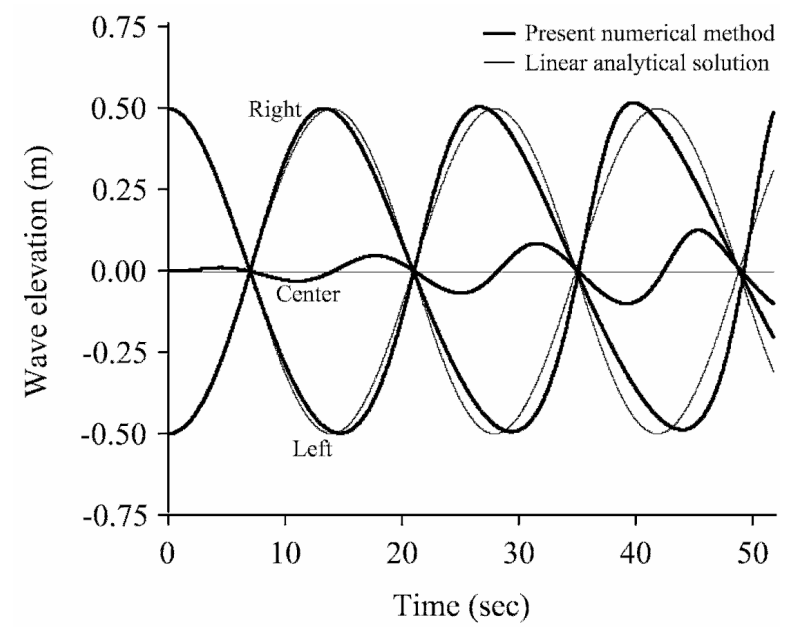

Figure 13. Surface wave elevation for $0.5 \mathrm{~m}$ amplitude.

In Figure 14 the time history of the wave elevation with amplitude of $2.0 \mathrm{~m}$ is presented. For such a large amplitude initial wave, the nonlinear effects appear clearly and the wave elevation arises to about $3.5 \mathrm{~m}$. Again comparison between the results and those in [8] shows the capability of the presented method in simulation of large amplitude waves. Figure 15 demonstrates the pressure contours and velocity vectors at different time steps; it also shows the free surface configuration at different time steps.

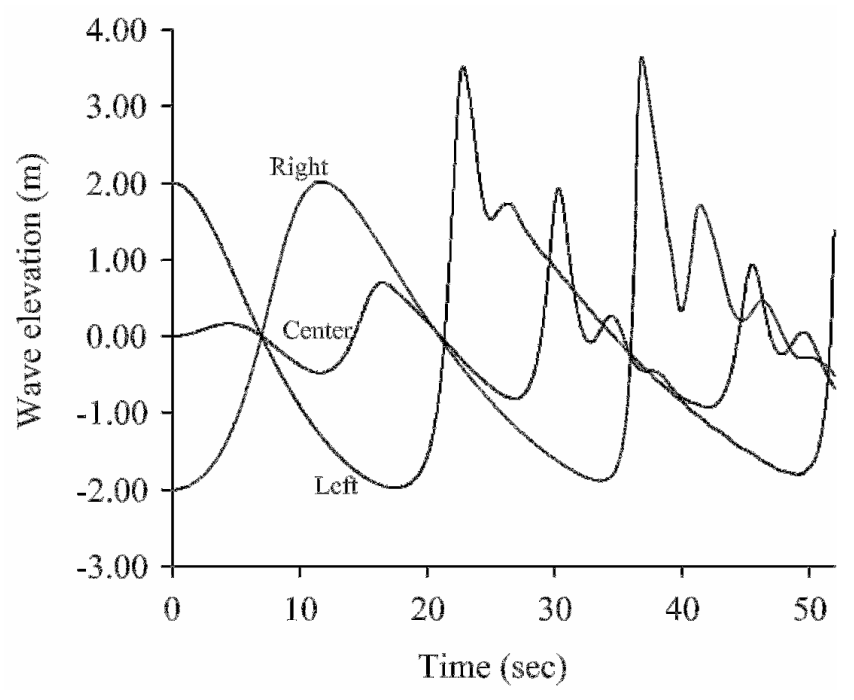

Figure 14. Surface wave elevation for $2.0 \mathrm{~m}$ amplitude.

\subsection{Solitary wave propagation}

Solitary wave propagation is a benchmark problem that is widely used for evaluating the capability of numerical methods in simulating free surface flows. Figure 16 shows the problem geometry and the definition of the parameters. A wave moves from the center of the reservoir and, after colliding with the right wall, travels back to its initial position. For such a problem experimental [31], analytical [32] and numerical $[6,8,9,10,33,34]$ results are available in the literature. 


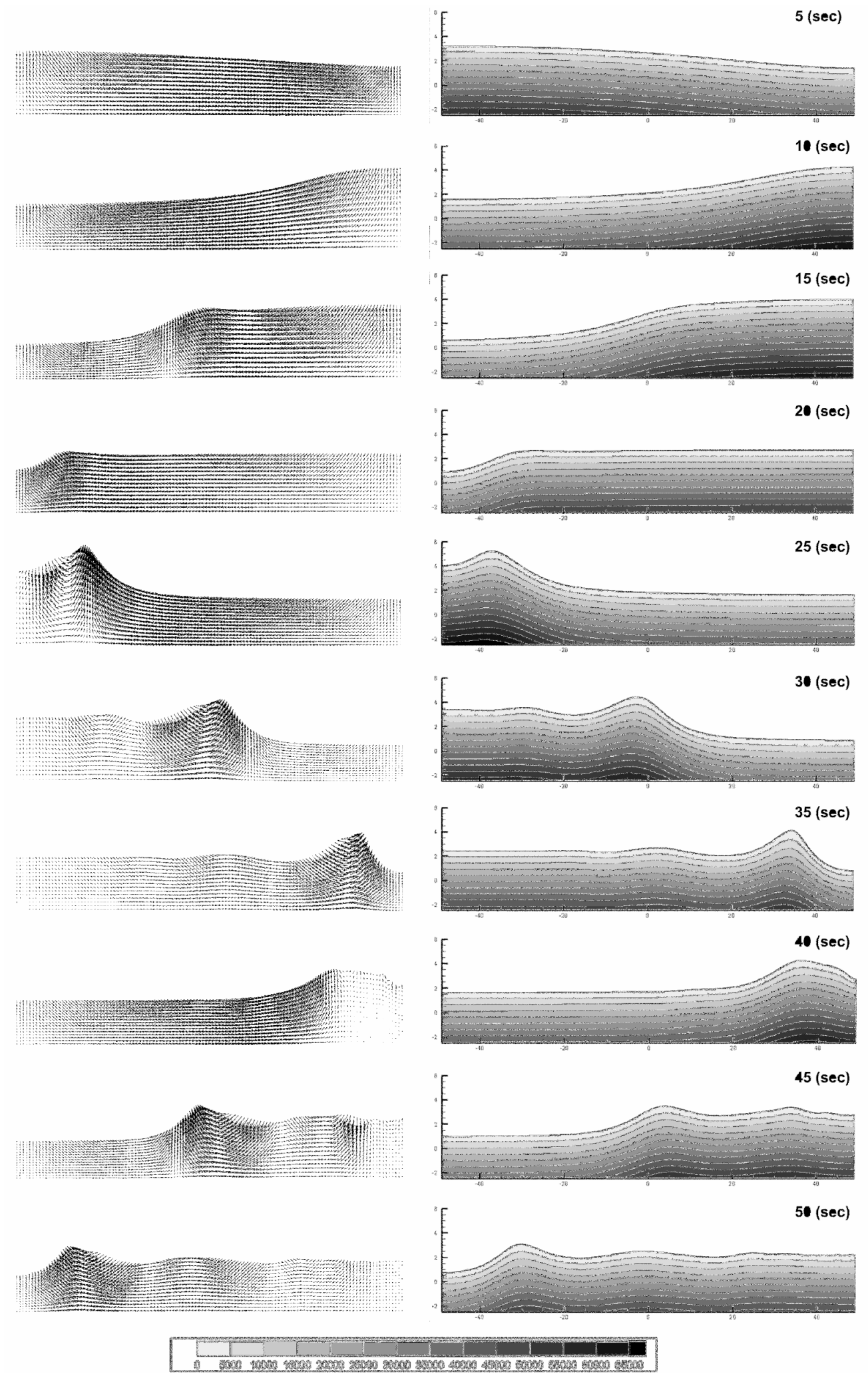

Figure 15. Pressure contours and velocity vectors of large amplitude oscillation in a rectangular basin, $\mathrm{A}=2.0 \mathrm{~m}$ (Example 4.3). 


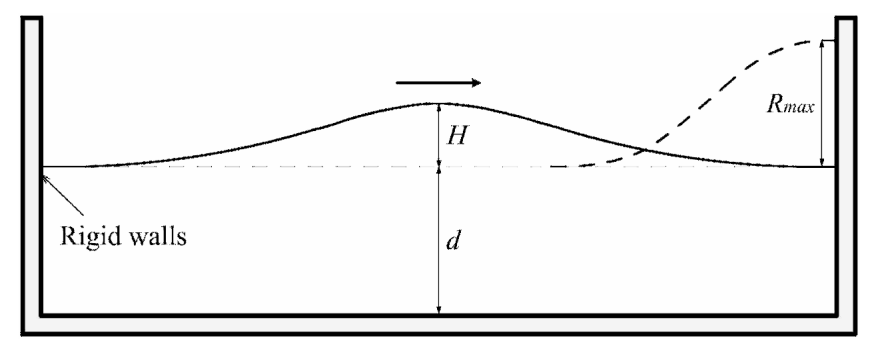

Figure 16. Solitary wave propagation: problem definition.

Total length of the basin is $L=16 d$ and $d=1$. The gravity acceleration is $g=9.81$ and the density of water is $\rho=1000$. The initial conditions are calculated according to the solution presented by Laitone [35] for an infinite domain. Based on Laitone's work, the total wave height, velocity components and pressure for a solitary wave propagating in an infinite domain are given as

$h=d+H \operatorname{sech}^{2}\left[\sqrt{\frac{3 H}{4 d^{3}}}(x-c t)\right]$,
$u=\sqrt{g d}+\frac{H}{d} \operatorname{sech}^{2}\left[\sqrt{\frac{3 H}{4 d^{3}}}(x-c t)\right]$,
$v=\sqrt{3 g d}+\left(\frac{H}{d}\right)^{3 / 2}\left(\frac{y}{d}\right) \operatorname{sech}^{2}\left[\sqrt{\frac{3 H}{4 d^{3}}}(x-c t)\right] \tanh \left[\sqrt{\frac{3 H}{4 d^{3}}}(x-c t)\right]$,

and

$p=\rho g(h-y)$,

in which $c$ satisfies the following relation

$\frac{c}{\sqrt{g d}}=1+\frac{1}{2} \frac{H}{d}-\frac{3}{20}\left(\frac{H}{d}\right)^{2}+O\left(\frac{H}{d}\right)^{3}$.

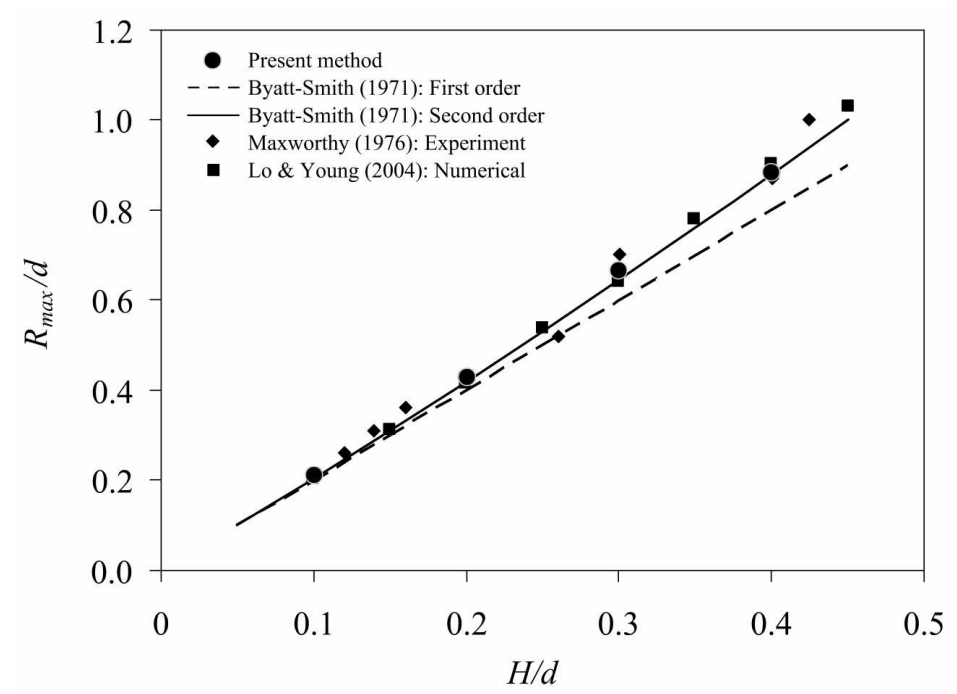

Figure 17. The maximum run-up height at the right wall versus initial wave height (Example 4.4). 
The initial conditions including velocity components and free surface position are obtained by substituting $t=0$ in Laitone's solution. The simulation was carried out for $H / d=0.1,0.2,0.3,0.4$ using 221 boundary points and 400 EBFs. It is worthwhile to mention that for a FEM simulation 16000 triangular elements with 4221 nodes are used in [8] and 3838 triangular elements with 2092 nodes are used in [8] for this problem. Time increment is set to $\Delta t=0.01 \mathrm{sec}$. Byatt-Smith [32] presented the following analytical relation for maximum run-up height at the right wall:

$$
\frac{R_{\max }}{d}=2\left(\frac{H}{d}\right)+\frac{1}{2}\left(\frac{H}{d}\right)^{2}+O\left(\frac{H}{d}\right)^{3},
$$

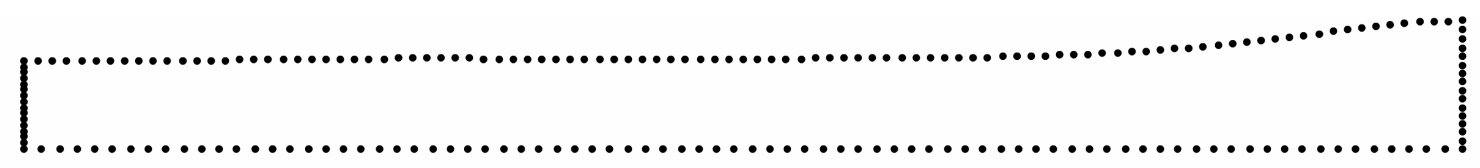

(a)

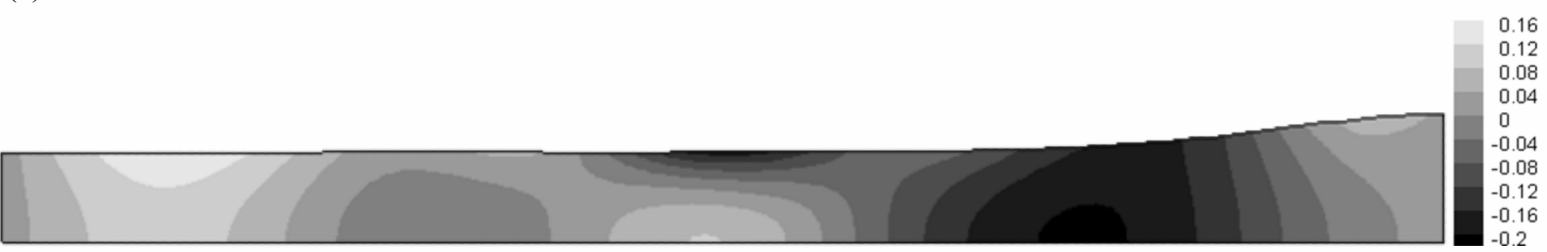

(b)

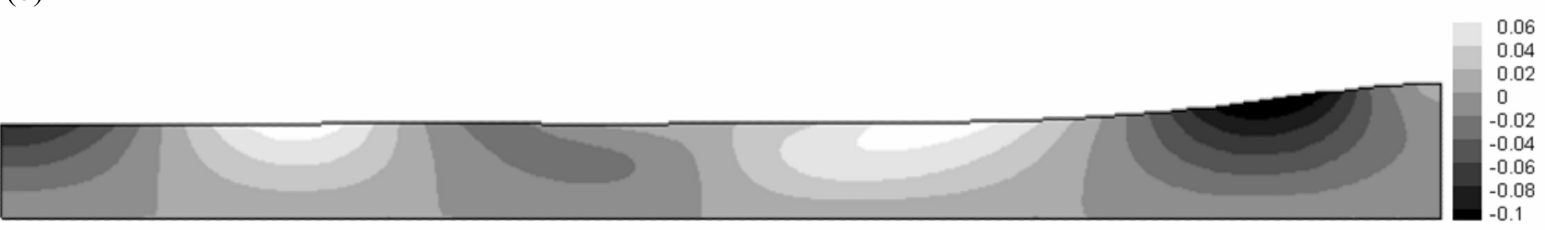

(c)

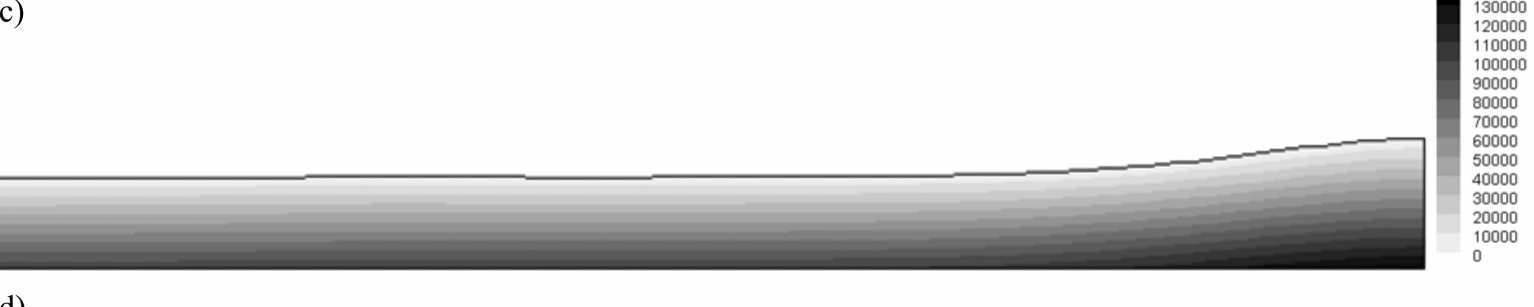

(d)

Figure 18. Solitary wave propagation (Example 4.4) with $\mathrm{H} / \mathrm{d}=0.2$ at time $\mathrm{t}=8.0$ (sec): (a) boundary points; (b) $\mathrm{u}$ $(\mathrm{m} / \mathrm{s}) ;(\mathrm{c}) \mathrm{v}(\mathrm{m} / \mathrm{s})$; and $(\mathrm{d}) \mathrm{p}(\mathrm{Pa})$.

The maximum run-up height at the right wall is shown in Figure 17. The numerical results are in close agreement with the analytical one proposed by Byatt-Smith and in all cases the differences are less than 3\% with the second order theory. In Figure 18, the boundary points, velocity, and pressure distribution are presented. Figure 19 illustrates the time history of the maximum wave height for the case of $H / d=0.2$ which is in excellent agreement with the results obtained in [9]. In this case, we have used $d=10$ for comparison. Figure 20 depicts the distribution of the vertical velocity for every $2.5 \mathrm{sec}$ when $H / d=0.3$. 


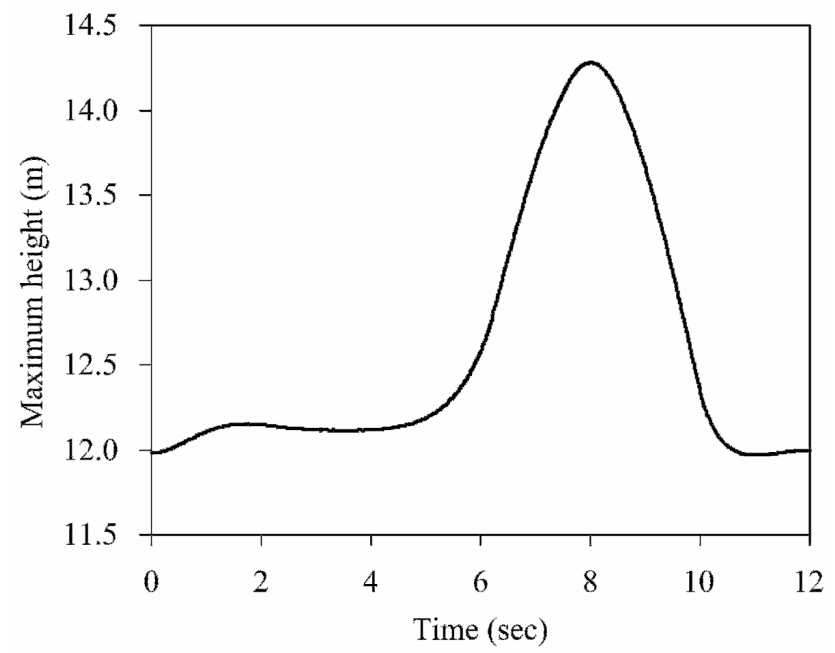

Figure 19. Time history of maximum wave height for $\mathrm{H} / \mathrm{d}=0.2$ and $\mathrm{d}=10(\mathrm{~m})$ (Example 4.4)
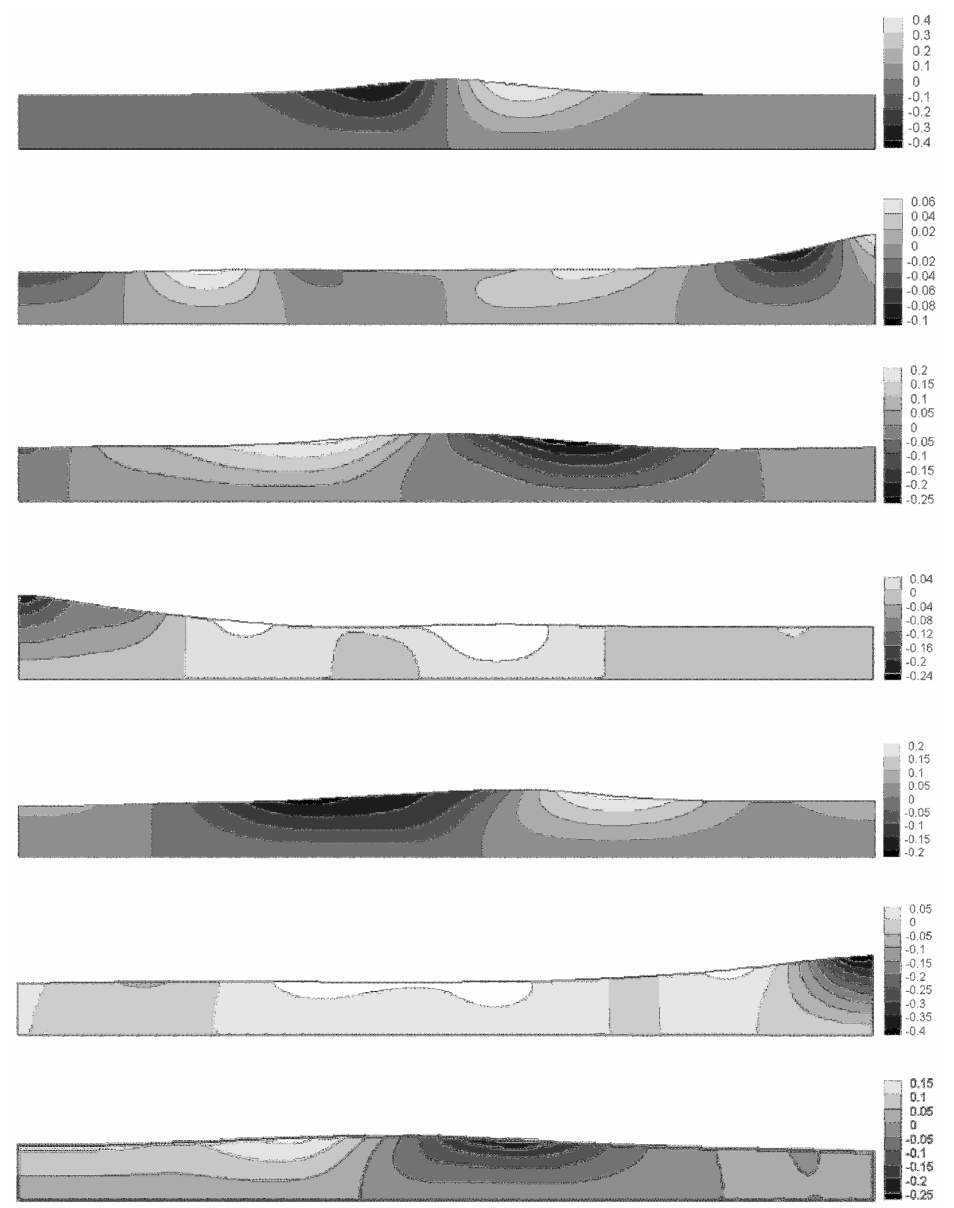

Figure 20. Vertical velocity distribution, Example 4.4, for every $2.5(\mathrm{sec})$ when $\mathrm{H} / \mathrm{d}=0.3$. 


\subsection{Interaction of two opposite solitary waves}

In this section, the collision of two opposite solitary waves with different amplitudes is simulated. The problem has been analytically and experimentally discussed in [31,36,37]. Also, it has been numerically simulated in [7] using ALE approach. In [37] the maximum run-up height during the collision has been estimated by the following relation

$H_{\max }=H_{L}+H_{R}+\frac{1}{2} H_{L} H_{R}+\frac{3}{8} H_{L} H_{R}\left(H_{L}+H_{R}\right)$,

in which $H_{L}$ and $H_{R}$ are the initial wave amplitudes on the left and right-hand sides, respectively.

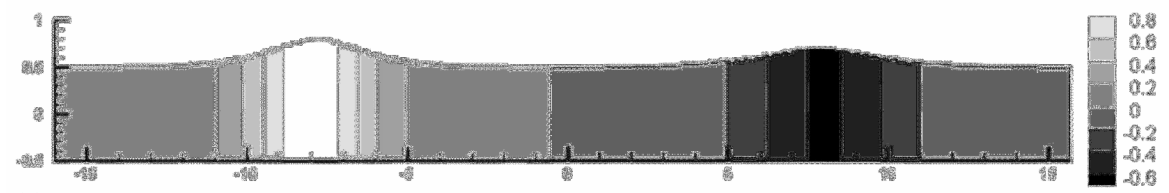

(a)

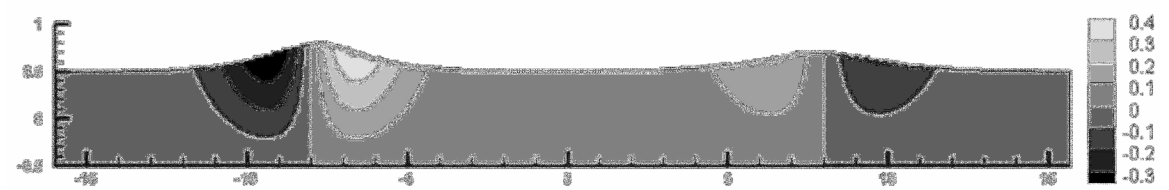

(b)

Figure 21. Initial velocity distributions for interaction of two opposite solitary waves, Example 4.5, for $\mathrm{H}_{\mathrm{L}}=0.3 \mathrm{~d}$ and $\mathrm{H}_{\mathrm{R}}=0.2 \mathrm{~d}:$ (a) $\mathrm{u}(\mathrm{m} / \mathrm{s}) ;(\mathrm{b}) \mathrm{v}(\mathrm{m} / \mathrm{s})$.

Here we consider a rectangular domain with length $L=32 d$ while $d=1$. Initial geometry and velocities are calculated using Laitone's solution. These initial conditions are demonstrated in Figure 21 for when the left-hand side amplitude is $H_{L}=0.3 d$ and the right-hand side amplitude is $H_{R}=0.2 d$. The simulation has been carried out using present method with 240 boundary points and 480 EBFs. The results of maximum run-up height for different amplitudes are tabulated in Table 1. Figure 22 shows velocity vectors at different time steps.

Table 1. Maximum run-up height for different amplitudes (Example 4.5)

\begin{tabular}{lccc}
\hline & $H_{L}=0.3 d, H_{R}=0.3 d$ & $H_{L}=0.3 d, H_{R}=0.2 d$ & $H_{L}=0.2 d, H_{R}=0.2 d$ \\
\hline Eq. (68) & 0.66525 & 0.54125 & 0.4260 \\
Present method & 0.64659 & 0.52902 & 0.4217 \\
\hline
\end{tabular}

\section{Conclusions}

In this report we presented a method in which a series of EBFs have been used to solve free surface flow through a Lagrangian description. The EBFs are found by defining characteristic equations from the governing differential equations in incompressible inviscid fluid flow problems. The boundary conditions are imposed through a collocation approach and thus the method can be categorized in meshless types. In the presented method, the number of EBFs does not need to be equal to that of the boundary information. A transformation technique has been employed for the evaluation of the unknown coefficients. 

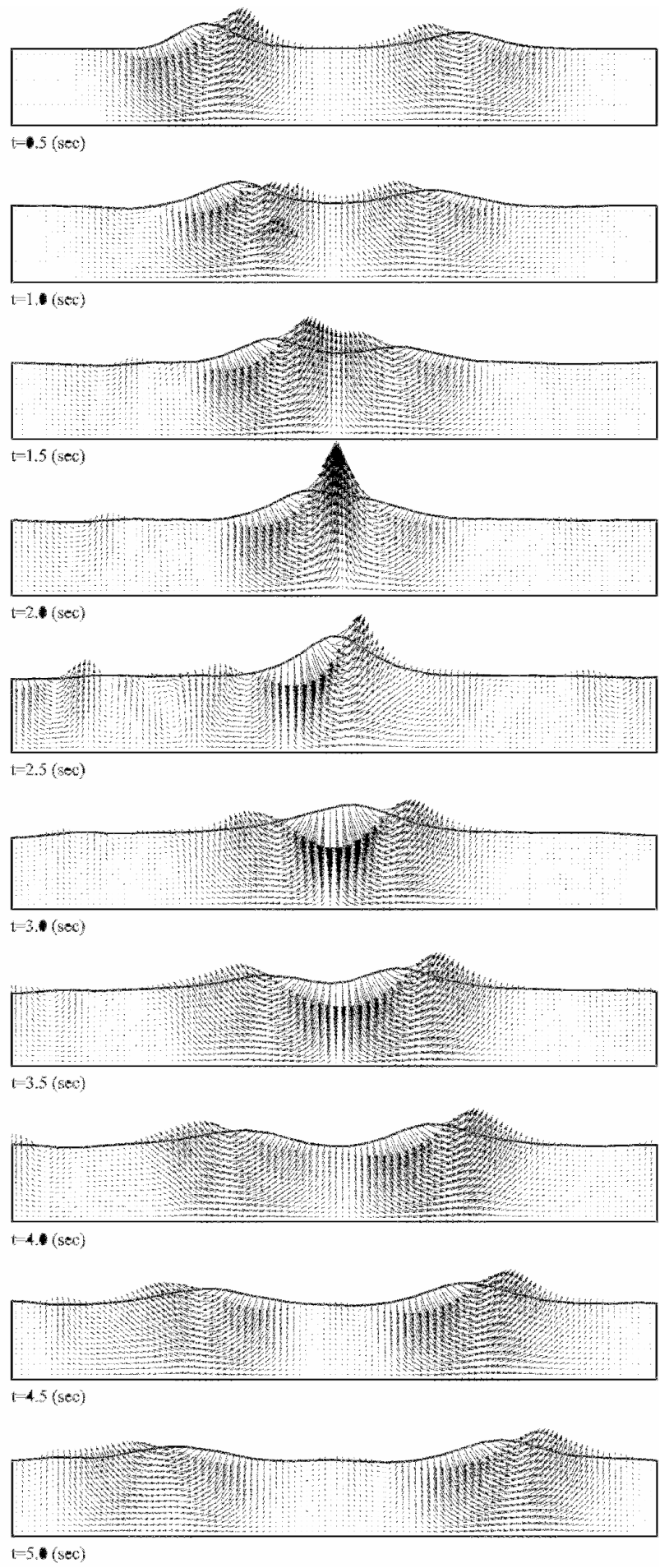

Figure 22. Velocity vectors for collision between two opposite solitary waves with $H_{L}=0.3 d$ and $H_{R}=0.2 d$.

The use of EBFs helps to find boundary velocities with high accuracy leading to a precise geometry updating. The developed Lagrangian meshless algorithm has been applied to variety of linear and nonlinear benchmark problems. Excellent agreement is seen between the obtained results 
and those available in the literature. The results show the capability of the method for simulating free surface flows.

The numerical method used for modeling of sloshing effect can easily be extended to investigate three-dimensional free surface flow problems. Our parallel researches show that one can solve Laplace equation in three-dimension with high accuracy. For solving such problems it is enough to replace the two dimensional EBFs with the three-dimensional ones in the explained procedure after defining the geometry, and its normal vectors to the surfaces, in three-dimensional space.

\section{Appendix A:}

The heuristic strategy for choosing $\alpha$ and $\beta$ proposed in [23] is as follows

$$
\alpha= \pm \frac{\bar{m} \bar{\gamma}}{\bar{L}}\left(\frac{\bar{k}}{\bar{N}}+2 \mathbf{i}\right), \quad \bar{m}=1, \ldots, \bar{M}, \quad \bar{k}=1, \ldots, \bar{N}
$$

for $\beta= \pm \mathbf{i} \alpha$. In a similar manner, when $\alpha= \pm \mathbf{i} \beta$ we select

$$
\beta= \pm \frac{\bar{m} \bar{\gamma}}{\bar{L}}\left(\frac{\bar{k}}{\bar{N}}+2 \mathbf{i}\right), \quad \bar{m}=1, \ldots, \bar{M}, \quad \bar{k}=1, \ldots, \bar{N}
$$

In the above relations, we choose $(\bar{M}, \bar{N}) \in \mathbb{N}^{2}, \bar{\gamma} \in \mathbb{R}$ and $\bar{L}$ is a characteristic length. The following bounds are found to be appropriate for many cases

$$
5.6 \leq \bar{\gamma} \leq 7.2(\text { say } \bar{\gamma}=2 \pi), \bar{L}=1.6 \max \left(L_{x}, L_{y}\right), \bar{M}_{\min }=4, \bar{N}_{\min }=2, \bar{N}_{\max }=8
$$

where $L_{x}$ and $L_{y}$ are the dimensions of the rectangle that circumscribes the domain. According to this pattern, the number of constructed EBFs is $8 \bar{M} \times \bar{N}$. For modelling of the problems presented in this report we have used $\bar{\gamma}=2 \pi, \bar{N}=5$ and $\bar{M}=10-12$. It should be noted that we calculate $L_{x}$ and $L_{y}$ at $t=0$.

\section{References}

1. OC. Zienkiewicz, RL. Taylor, The finite element method, Vol. 3 (5th edn), Butterworth-Heineman, 2000.

2. B. Ramaswamy, M. Kawahara, Lagrangian finite element method for the analysis of two-dimensional sloshing problems, International Journal for Numerical Methods in Fluids 6 (1986) 659-670.

3. B. Ramaswamy, M. Kawahara, Lagrangian finite element analysis applied to viscous free surface fluid flow, International Journal for Numerical Methods in Fluids 7 (1987) 953-984.

4. R. Radovitzky, M. Oritz, Lagrangian finite element analysis of Newtonian fluid flow, International Journal for Numerical Methods in Engineering 43 (1998) 607-619.

5. S.R. Idelsohn, E. Oñate, F. Del Pin, A Lagrangian meshless finite element method applied to fluid-structure interaction problems, Computers and Structures 81 (2003) 655-671.

6. B. Ramaswamy, M. Kawahara, Arbitrary Lagrangian-Eulerian finite element method for unsteady, convective, incompressible viscous free surface fluid flow, International Journal for Numerical Methods in Fluids 7 (1987) 1053-1075.

7. S. Ushijima, Three-dimensional arbitrary Lagrangian-Eulerian prediction method for non-linear free surface oscillation, International Journal for Numerical Methods in Fluids 26 (1998) 605-623.

8. D.C. Lo, D.L. Young, Arbitrary Lagrangian-Eulerian finite element analysis of free surface flow using a velocityvorticity formulation, Journal of Computational Physics 195 (2004) 175-201. 
9. F. Durate, R. Gormaz, S. Natesan, Arbitrary Lagrangian-Eulerian method for Navier-Stokes equations with moving boundaries, Computer Methods in Applied Mechanics and Engineering 193 (2004) 4819-4836.

10. P. Nithiarasu, An arbitrary Lagrangian Eulerian (ALE) formulation for free surface flows using the characteristicbased split (CBS) scheme, International Journal for Numerical Methods in Fluids 48 (2005) 1415-1428.

11. P.H. Saksono, W.G. Dettmer, D. Perić, An adaptive remeshing strategy for flows with moving boundaries and fluidstructure interaction, International Journal for Numerical Methods in Engineering 71 (2007) 1009-1050.

12. R.A. Gingold, J.J. Monaghan, Smoothed particle hydrodynamics, theory and application to non-spherical stars, Monthly Notices of the Royal Astronomical Society 181 (1997) 375 -389.

13. S. Koshizuka, Y. Oka, Moving particle semi-implicit method for fragmentation of incompressible fluid, Nuclear Engineering Science 123 (1996) 421- 434.

14. Y. Suzuki, S. Koshizuka, Y. Oka, Hamiltonian moving-particle semi-implicit (HMPS) method for incompressible fluid flows, Computer Methods in Applied Mechanics and Engineering 196 (2007) 2876-2894.

15. J. Bonet, S. Kulasegaram, Correction and stabilization of smooth particle hydrodynamics methods with applications in metal forming simulation, International Journal for Numerical Methods in Engineering 47 (2000) 1189-1214.

16. S.R. Idelsohn, E. Oñate, F. Del Pin, The particle finite element method: a powerful tool to solve incompressible flows with free surfaces and breaking waves, International Journal for Numerical Methods in Engineering 61 (2004) 964-989.

17. E. Oñate, S.R. Idelsohn, O.C. Zienkiewicz, R.L Taylor, A finite point method in computational mechanics. Applications to convective transport and fluid flow, International Journal for Numerical Methods in Engineering 39 (22) (1996) $3839-3886$.

18. E. Oñate, S.R. Idelsohn, O.C. Zienkiewicz, R.L Taylor, C. Sacco, A stabilized finite point method for analysis of fluid mechanics problems, Computer Methods in Applied Mechanics and Engineering 39 (1996) 315-346.

19. E. Kita, J. Katsuragawa, N. Kamiya, Application of Trefftz-type boundary element method to simulation of twodimensional sloshing phenomenon, Engineering Analysis with Boundary Elements 28 (2004) 677-683.

20. B. Boroomand, F. Mossaiby, Generalization of robustness test procedure for error estimators, part I: formulation for patches near kinked boundaries, International Journal for Numerical Methods in Engineering 64 (2005) 427-460.

21. B. Boroomand, F. Mossaiby, Generalization of robustness test procedure for error estimators, part II: test results for error estimators using SPR and REP, International Journal for Numerical Methods in Engineering 64 (2005) 461502.

22. B. Boroomand, F. Mossaiby, Dynamic solution of unbounded domains using finite element method: discrete Green's functions in frequency domain, International Journal for Numerical Methods in Engineering 67 (2006) 1491-1530.

23. B. Boroomand, S. Soghrati, B. Movahedian, Exponential basis functions in solution of static and time harmonic elastic problems in a meshless style, International Journal for Numerical Methods in Engineering 81 (2010) 9711018.

24. S.M. Zandi, B. Boroomand, Two-dimensional incompressible materials analysis using standard formulation in a meshless style, Proceedings of the Seventh International Conference on Engineering Computational Technology (ECT 2010), Valencia, Spain, September 2010. doi:10.4203/ccp.94.117.

25. Z.C. Li, T.T. Lu, H.T. Huang, A.H.D. Cheng, Trefftz, collocation, and other boundary methods-A comparison, Numerical Methods for Partial Differential Equations 23 (2007) 93-144.

26. B.F. Chen, R. Nokes, Time-independent finite difference analysis of fully non-linear and viscous fluid sloshing in a rectangular tank, Journal of Computational Physics 209 (2005) 47-81.

27. Y.H. Chen, W.S. Hwang, C.H. Ko, Sloshing behaviours of rectangular and cylindrical liquid tanks subjected to harmonic and seismic excitations, Earthquake Engineering and Structural Dynamics 36 (2007) 1701-1717.

28. R.A. Ibrahim, V.N. Pilipchuk, T. Ikeda, Recent advances in liquid sloshing dynamics, Applied Mechanics Reviews 54 (2) (2001) 133-199.

29. G.X. Wu, R. Eatock Taylor, Finite element analysis of two dimensional non-linear transient water waves, Applied Ocean Research 16 (1994) 363-372.

30. G. Neumann, W.J. Pierson, Principle of physical oceanography, Prentice-Hall, 1966.

31. T. Maxworthy, Experiments on collisions between solitary waves, Journal of Fluid Mechanics 76 (1976) 177-185.

32. J.G.B. Byatt-Smith, An integral equation for unsteady surface waves and a comment on the Boussinesq equation, Journal of Fluid Mechanics 49 (1971) 625-633.

33. D. González, E. Cueto, F. Chinesta, M. Doblaré, A natural element updated Lagrangian strategy for free-surface fluid dynamics, Journal of Computational Physics 223 (2007) 127-150.

34. M.S. Kim, W.I. Lee, A new VOF-based numerical scheme for the simulation of fluid flow with free surface. Part I: New free surface-tracking algorithm and its verification, International Journal for Numerical Methods in Fluids 42 (2003) 765-790. 
35. E.V. Laitone, The second approximation to cnoidal and solitary waves, Journal of Fluid Mechanics 9 (1960) 430444.

36. C.H. Su, R.M. Mirie, On head-on collision between two solitary waves, Journal of Fluid Mechanics 98 (1980) 509525.

37. R.M. Mirie, C.H. Su, Collisions between two solitary waves. Part 2: A numerical study, Journal of Fluid Mechanics 115 (1982) 475-492.

\section{List of Figures}

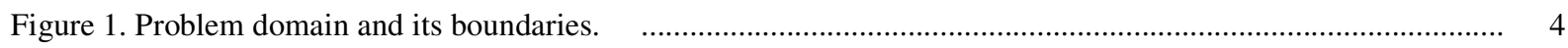

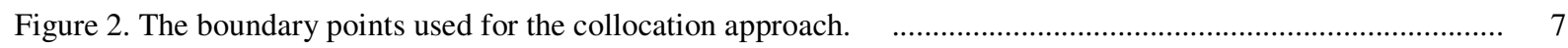

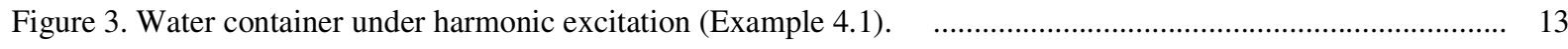

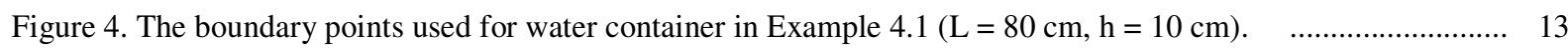

Figure 5. The results obtained for the rectangular tank (Example 4.1) subjected to harmonic excitation with forced

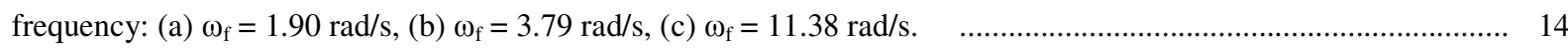

Figure 6. Free surface profiles of the rectangular tank (Example 4.1) subjected to harmonic excitation with forced

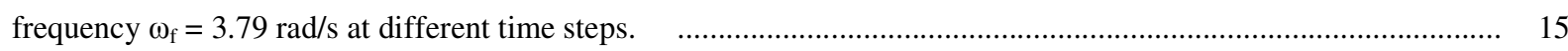

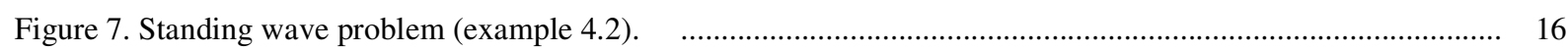

Figure 8. Initial free surface profile and boundary points used for standing wave problem (Example 4.2). $\quad$........... 16

Figure 9. Comparison of the calculated wave heights at the center of the tank (Example 4.2). $\quad$............................... 17

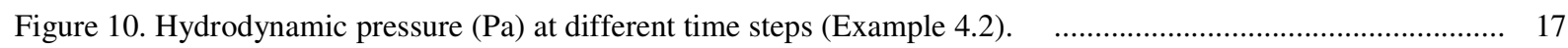

Figure 11. Rectangular basin with a half cosine wave (Example 4.3). ….................................................... 18

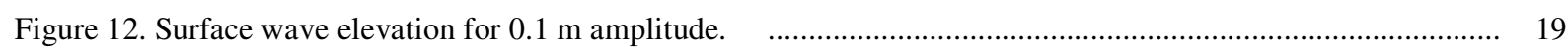

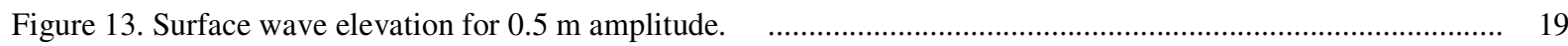

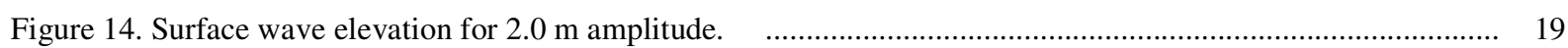

Figure 15. Pressure contours and velocity vectors of large amplitude oscillation in a rectangular basin, A=2.0 $\mathrm{m}$

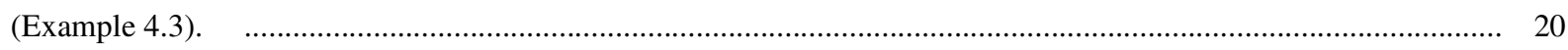

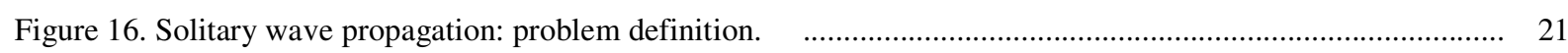

Figure 17. The maximum run-up height at the right wall versus initial wave height (Example 4.4). $\quad \ldots \ldots \ldots \ldots \ldots \ldots \ldots . . . . . . . . . .21$

Figure 18. Solitary wave propagation (Example 4.4) with $\mathrm{H} / \mathrm{d}=0.2$ at time $\mathrm{t}=8.0$ (sec): (a) boundary points; (b) $\mathrm{u}$ $(\mathrm{m} / \mathrm{s}) ;(\mathrm{c}) \mathrm{v}(\mathrm{m} / \mathrm{s})$; and $(\mathrm{d}) \mathrm{p}(\mathrm{Pa})$.

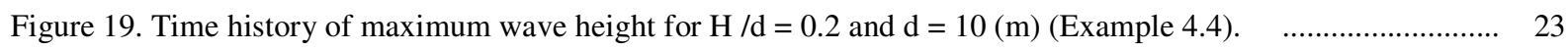

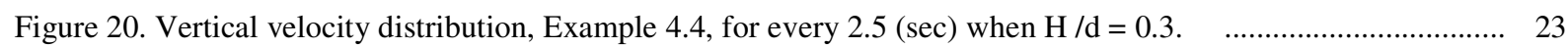

Figure 21. Initial velocity distributions for interaction of two opposite solitary waves, Example 4.5 , for $\mathrm{H}_{\mathrm{L}}=0.3 \mathrm{~d}$ and

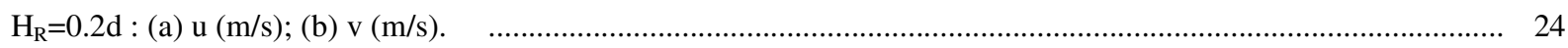

Figure 22. Velocity vectors for collision between two opposite solitary waves with $\mathrm{H}_{\mathrm{L}}=0.3 \mathrm{~d}$ and $\mathrm{H}_{\mathrm{R}}=0.2 \mathrm{~d}$. $\quad \ldots \ldots \ldots \ldots$

\section{List of Tables}

Table 1. Maximum run-up height for different amplitudes (Example 4.5). 